\title{
An automatic approach to exclude interlopers from asteroid families
}

\author{
Viktor Radović, ${ }^{1 \star}$ Bojan Novaković, ${ }^{1}$ Valerio Carruba ${ }^{2}$ and Dušan Marčeta ${ }^{1}$ \\ ${ }^{1}$ Department of Astronomy, Faculty of Mathematics, University of Belgrade, Studentski trg 16, 11000 Belgrade, Serbia \\ ${ }^{2}$ UNESP, Univ. Estadual Paulista, Grupo de dinamica Orbital e Planetologia, Guaratinguet, SP 12516-410, Brazil
}

Accepted 2017 May 19. Received 2017 May 19; in original form 2017 March 21

\begin{abstract}
Asteroid families are a valuable source of information to many asteroid-related researches, assuming a reliable list of their members could be obtained. However, as the number of known asteroids increases fast it becomes more and more difficult to obtain a robust list of members of an asteroid family. Here, we are proposing a new approach to deal with the problem, based on the well-known hierarchical clustering method. An additional step in the whole procedure is introduced in order to reduce a so-called chaining effect. The main idea is to prevent chaining through an already identified interloper. We show that in this way a number of potential interlopers among family members is significantly reduced. Moreover, we developed an automatic online-based portal to apply this procedure, i.e. to generate a list of family members as well as a list of potential interlopers. The Asteroid Families Portal is freely available to all interested researchers.
\end{abstract}

Key words: minor planets, asteroids, general.

\section{INTRODUCTION}

Asteroid families are formed when a collision breaks apart a parent body into numerous smaller fragments (Zappala et al. 1984). As such, they are very important for almost any asteroid-related research. For example, families may provide us a clue about collisional history of the main asteroid belt (see e.g. Cibulková, Brož \& Benavidez 2014, and references therein), asteroid interiors (Cellino et al. 2002), as well as space weathering effects that alter asteroids colour with time (Nesvorný et al. 2005). Moreover, they may even tell us about the origins of some near-Earth asteroids (e.g. Walsh et al. 2013; Bottke et al. 2015; Novaković et al. 2017), main-belt comets (Novaković et al. 2014; Hsieh et al. 2015) or help us put constraints on some past phenomena like the Late Heavy Bombardment (Brož et al. 2013).

The impact ejects fragments away from the parent body at velocities similar to the parent's body escape speed, which is typically not more than a few tens of metres per second. In the main asteroid belt, these velocities are much slower than the orbital ones, which are about $15-20 \mathrm{~km} \mathrm{~s}^{-1}$. Therefore, the impact-produced objects initially keep orbits similar to the orbit of their parent body. This fact allows identification of the so-called dynamical asteroid families, i.e. groups of asteroids identified based on the similarity of their orbital parameters. Still, from the orbital similarity point of view, it is important to distinguish young asteroid families from older ones.

Very young families $\left(t_{\text {age }}<1 \mathrm{Myr}\right)$ may be still recognized in the space of instantaneous (osculating) orbital elements, because dif-

^E-mail: rviktor@matf.bg.ac.rs ferent perturbations have not yet had enough time to disperse orbits of their members (Nesvorný \& Vokrouhlický 2006). On the other hand, older families are usually identified using the so-called proper orbital elements that are nearly constant over time (Knežević \& Milani 2000).

So far a large number of asteroid families have been discovered across the main asteroid belt (e.g. Zappala et al. 1990; Nesvorný et al. 2005; Novaković, Cellino \& Knežević 2011; Masiero et al. 2013; Milani et al. 2014, 2017; Nesvorný, Brož \& Carruba 2015). These families offer a wide range of possibilities for further studies, yet an essential prerequisite for all these studies is a reliably established family membership. However, as nowadays the number of known asteroids increases fast, it becomes more and more difficult to obtain a robust list of members of an asteroid family.

For the purpose of family identification the hierarchical clustering method (HCM), proposed by Zappala et al. (1990), is most widely used. ${ }^{1}$ It connects all objects whose mutual distances in the threedimensional proper-element space are below a threshold value.

The HCM, however, obviously has some limitations in distinguishing between real family members and nearby background asteroids. $^{2}$ This gives rise to the well-known problem of presence

\footnotetext{
${ }^{1}$ Besides the HCM, the wavelet analysis method (WAM) was also successfully applied to identify asteroid families (Bendjoya, Slezak \& Froeschle 1991).

${ }^{2}$ This problem is not due to the HCM itself, and is not limited to this method only. For instance, the WAM suffers from the same problem (Zappalà et al. 1995).
} 
of interlopers among the members of an asteroid family (Migliorini et al. 1995).

A possible way to deal with this problem is to use additional information to discriminate real family members from interlopers. Asteroids that belong to the same family generally have similar mineral composition (Cellino et al. 2002). Thus, data about their spectra/colours or albedos may help to determine membership of an asteroid family more reliably.

This additional information may be used in different ways. One possible method to exploit available physical data is to apply the HCM in extended space, i.e. in the space that also includes physical data in addition to the three proper elements. Parker et al. (2008) applied the HCM in four-dimensional space, using the Sloan Digital Sky Survey (SDSS; York et al. 2000) colours as the fourth dimension. Carruba et al. (2013) extend this approach to the fifth dimension using albedos as an additional dimension, significantly reducing the percentage of known interlopers with respect to other methods.

Another possible procedure is to first separate the main-belt asteroids into two populations (typically representing $C$ - and $S$-type objects) according to their colour and albedo values. The HCM is then applied to each of these populations separately (Masiero et al. 2013). In this way, Masiero et al. (2013) managed to identify several new families.

These methods, despite being in general very efficient, have a serious limitation that they can only be applied to a reduced set of main-belt asteroids for which the colours and albedos are obtained. Despite significant increase of available physical data in the recent years, the number of asteroids for which these data are at our disposal is still several times smaller than the number of objects for which proper elements have been computed. As explained by Milani et al. (2014), the dynamical parameters, in this case the proper elements, have a larger information content than the physical observations, because the latter are available either for significantly smaller catalogues, or with lower relative accuracy.

For these reasons, some authors adopted a bit different approach, so that the so-called dynamical families are first obtained in the space of proper elements, and available physical data are used only posteriori to distinguish possible overlapping families or to identify interlopers among family members (see e.g. Novaković et al. 2011; Milani et al. 2014, 2016).

All the above-described methodologies are useful to some extent. Still, they have some limitations imposed by the HCM itself. A wellknown drawback of the HCM based on the single linkage rule is the so-called chaining phenomenon; that is, first concentrations tend to incorporate nearby groups, forming as a result a kind of chain that may consist of non-family members (Zappala et al. 1994).

In this paper we extend previous works in two directions:

(i) First, we are introducing an additional step in the whole procedure, aiming mainly to reduce the chaining effect. The main idea is that if an interloper is identified as a family member, it may cause more interlopers to be added due to the chaining effect. In this manner we managed to further reduce the number of potential interlopers among family members, by preventing chaining through an already identified interloper.

(ii) Secondly, we are presenting here an automatic, free, onlinebased tool to apply our procedure. It allows us to generate a list of family members as well as a list of potential interlopers along with criterion for their rejection. This is important because despite numerous papers dealing with family members' identification and removal of potential interlopers, there is still a lack of publicly available information on these.

\section{METHOD}

The approach that we are using here is essentially very similar to methods that first identify dynamical families using only the proper orbital elements, and then apply physical data to further refine family membership. The only exception in this respect is an additional stage. The whole procedure could be divided in four main steps (Fig. 1):

(i) In the first step, the HCM analysis is performed using the whole (initial) catalogue of proper elements in order to obtain a preliminary list of family members.

(ii) In the second step, physical and spectral properties are used in order to identify interlopers among asteroids initially linked to a family.

(iii) In the third step, objects identified as interlopers in the second step are excluded from the initial catalogue of proper elements, producing a modified catalogue

(iv) Finally, in the fourth step, the HCM analysis is performed again, but this time using the reduced (modified) catalogue of proper elements, produced in the third step.

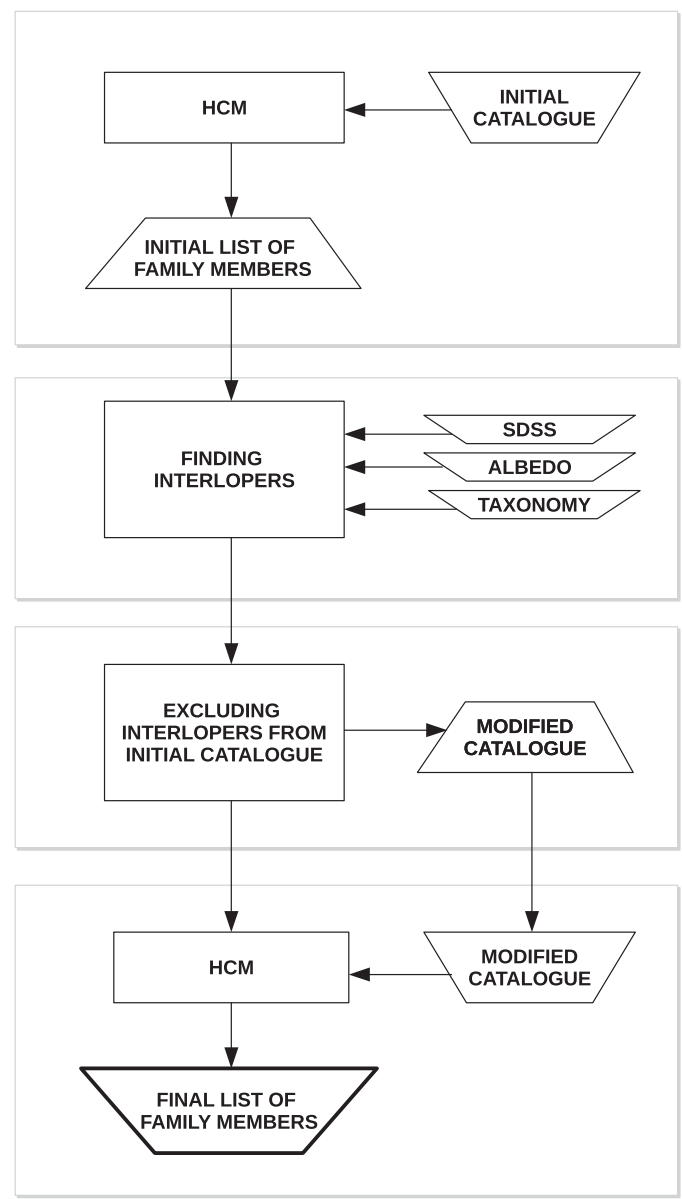

STEP 1

STEP 2

STEP 3

Figure 1. The chart showing the algorithm of our methodology to remove interlopers from asteroid families. 


\subsection{Step 1: obtaining a preliminary membership}

To obtain a preliminary list of family members, we apply the HCM in the space of three proper orbital elements ${ }^{3}$ : semimajor axis $a_{\mathrm{p}}$, eccentricity $e_{\mathrm{p}}$ and sine of inclination $\sin \left(i_{\mathrm{p}}\right)$. The HCM identifies an asteroid as a part of family if its distance $d$ from the closest neighbour is smaller than an adopted cut-off distance $d_{\text {cut }}$. The common definition of this metric is (Zappala et al. 1990)

$d=n a_{\mathrm{p}} \sqrt{C_{a}\left(\delta a_{\mathrm{p}} / a_{\mathrm{p}}\right)^{2}+C_{e}\left(\delta e_{\mathrm{p}}\right)^{2}+C_{i}\left(\delta \sin \left(i_{\mathrm{p}}\right)\right)^{2}}$,

where $n a_{\mathrm{p}}$ is the heliocentric orbital velocity of an asteroid on a circular orbit having the semimajor axes $a_{\mathrm{p}}, \delta a_{\mathrm{p}}=a_{\mathrm{p} 1}-a_{\mathrm{p} 2}, \delta e_{\mathrm{p}}=$ $e_{\mathrm{p}_{1}}-e_{\mathrm{p}_{2}}$ and $\delta \sin \left(i_{\mathrm{p}}\right)=\sin \left(i_{\mathrm{p}_{1}}\right)-\sin \left(i_{\mathrm{p}_{2}}\right)$, where the indexes (1) and (2) denote the two bodies whose mutual distance is calculated. The distance $d$ is expressed in metres per second.

The above metric is derived based on the well-known relationship between the components of ejection velocities of the fragments and the resulting differences in their orbital elements (Gaussian equations). For the constants, the most frequently used values are $C_{a}=5 / 4, C_{e}=2$ and $C_{i}=2$ (Zappala et al. 1994), but other values yield the similar results (Nesvorný et al. 2005).

Here, we applied the HCM around a selected central asteroid. In this case, the volume of the proper elements space is not fixed a priory, but it grows around the central object as the velocity cut-off distance increases. In order to define a preliminary list of family members, we need to adopt an appropriate value of cut-off distance $d_{\text {cut }}$. Generally, this is the distance in the space of proper elements that best describes a family. However, for a preliminary definition of the family our aim is slightly different. As we want to identify as many potential interlopers as possible, we used the largest reasonable value of $d_{\text {cut }}$, rather than the most appropriate one.

Thus, in the first step we derive the cut-off value as follows. Starting from the lowest value of $10 \mathrm{~m} \mathrm{~s}^{-1}$, we increased $d_{\text {cut }}$ by fixed step of $5 \mathrm{~m} \mathrm{~s}^{-1}$, until the family merges with a local background population of asteroids. For the threshold value we adopt one of the two steps $\left(10 \mathrm{~m} \mathrm{~s}^{-1}\right)$ below the distance at which the family merges with the background.

As mentioned above, the cut-off value obtained in this way is often too large to define a nominal family. In some cases this approach caused two (or even more) nearby families to merge into single group (see Sections 3.4 and 3.3 for discussion on these cases). Still, we use it in order to get as many family members (and consequently also interlopers) as possible.

\subsection{Step 2: interlopers identification}

Once a preliminary list of family members is obtained, the next step is the identification of potential interlopers among them. In this respect, let us recall the well-known fact that the members of a collisional family typically share similar physical and spectral characteristics (Bus 1999; Lazzaro et al. 1999; Cellino et al. 2002; Ivezić et al. 2002). Therefore, different spectral and photometric data could be used to complement the results of the HCM analysis, in order to obtain a list of potential interlopers.

Available observational data are often good enough only to distinguish between $C$ and $S$ classes of asteroids, while other taxonomy classes could not be separated reliably. An obvious exception is

\footnotetext{
${ }^{3}$ The catalogue of synthetic proper elements is available at AstDys web page: http://hamilton.dm.unipi.it.
}

spectral data that are, however, available for a very limited number of asteroids. A finer classification may also be possible to achieve with an advance classification algorithm developed by DeMeo \& Carry (2013), based on the SDSS data. Still, to stay on the safe side, we decided to resolve here only two aforementioned broad asteroid classes. This analysis is performed using SDSS colours, geometric albedos from different surveys and available spectroscopic data. Then, if it is found that a family is mostly composed of $C$-type asteroids, all potential members that belong to $S$ type are defined as interlopers, and vice versa.

\subsubsection{The Sloan Digital Sky Survey data}

The SDSS survey used five-colour photometric system $u, g, r, i$ and $z$, with central wavelengths of $0.3543,0.4770,0.6231,0.7625$ and $0.9134 \mu \mathrm{m}$, respectively. The fourth release of the SDSS Moving Object Catalogue (SDSS MOC) contains photometric data for 471569 moving objects observed up to 2007 March. Among these data, 220101 entries were successfully linked to 104449 unique moving objects.

It was shown by Ivezić et al. (2002) that SDSS photometry is consistent with available spectra of asteroids, meaning that the colours provided by the SDSS could be used to separate at least broad taxonomic classes, such as $C$ and $S$. This was also confirmed by Nesvorný et al. (2005) and Parker et al. (2008), who used the third and fourth releases of the SDSS MOC, respectively. In both cases, authors used a principal component analysis approach to link SDSS colours to spectral types. The main difference between the methods utilized by Nesvorný et al. (2005) and Parker et al. (2008) is that latter authors excluded $u$ band from their analysis, due to the large noise in $u$ band presented in the fourth version of SDSS MOC.

Moreover, Carvano et al. (2010) defined a new classification algorithm based on the SDSS colours that can be used to assign taxonomic classes to SDSS observations. This scheme is compatible with the Bus taxonomy and allows finer distinction between taxonomic classes than methodology used by Nesvorný et al. (2005) or Parker et al. (2008).

For our purpose here, we chose to use the fourth release of the SDSS MOC to distinguish between $C$ and $S$ taxonomic complexes. Thus, we adopt the Parker et al. (2008) approach excluding $u$ band from our analysis and using $a^{*}$ colour defined by Ivezić et al. (2001) as

$a^{*}=0.89(g-r)+0.45(r-i)-0.57$.

It is known that asteroids show bimodal distribution in $a^{*}$, where $C$-type objects are characterized with $a^{*}<0$, while $S$-type objects typically have $a^{*}>0$.

Being interested here in identifying potential interlopers among members of an asteroid family we proceed in the following way. First, for each family, we calculate the mean value $a_{\mathrm{m}}^{*}$ and its corresponding standard deviation $\sigma\left(a_{\mathrm{m}}^{*}\right)$, using colours of potential family members. The mean value is then used to define the spectral class of the family. Finally, all potential family members having their individual $a^{*}$ colour inconsistent with the one derived for the family are classified as interlopers. In order to keep the interloper identification as reliable as possible we introduce a safety margin between two types, based on the value of standard deviation of $a^{*}$ colour of an asteroid $\sigma\left(a^{*}\right)$. For instance, if an asteroid family is classified as $C$ type, only objects having $a^{*}<-3 \sigma\left(a^{*}\right)$ are considered to be interlopers, and likewise, if a family is $S$ type then objects 

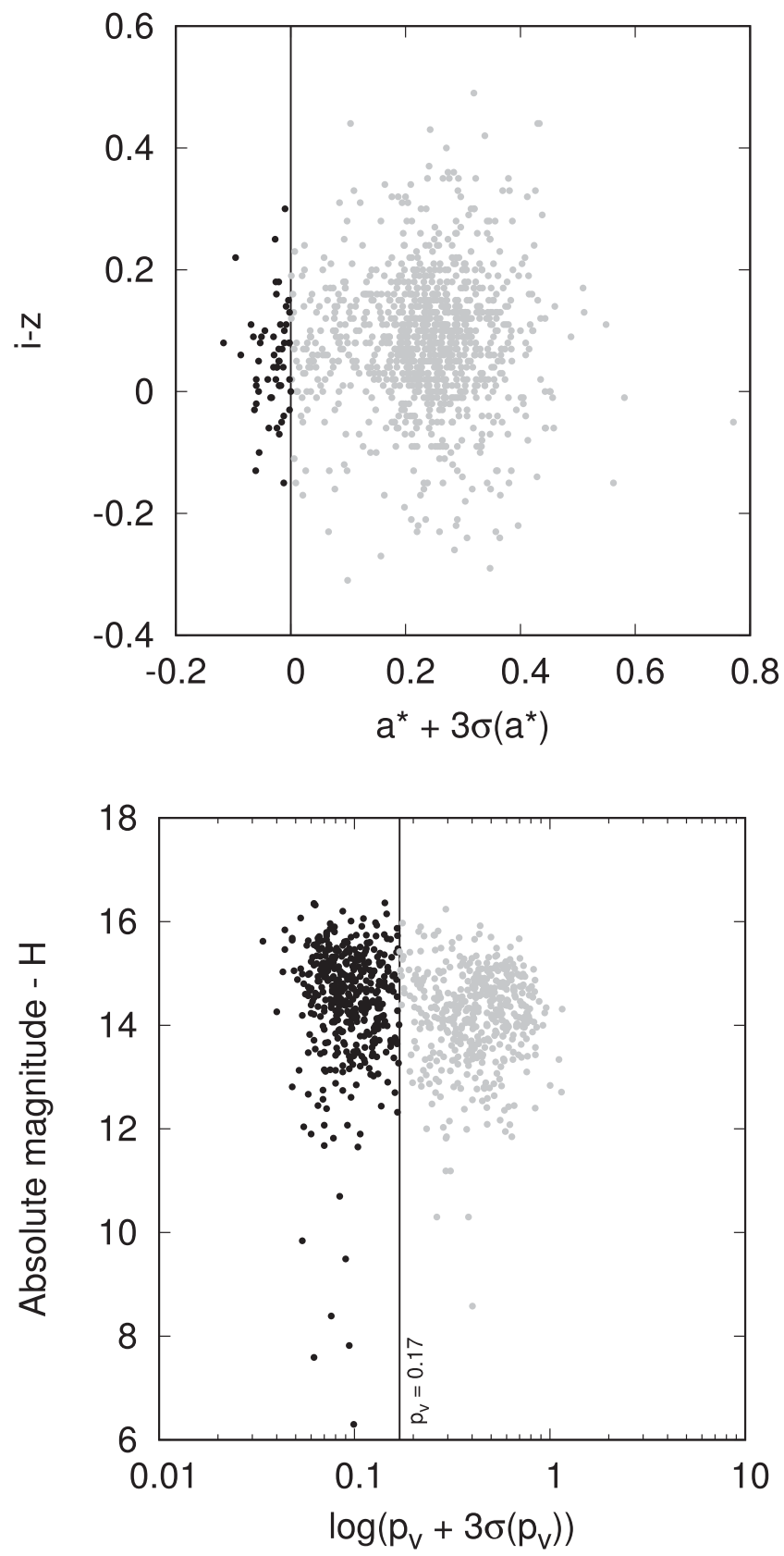

Figure 2. Identification of interlopers among the Klumpkea family members based on the SDSS colour (top panel) and albedo (bottom panel) data. Black and grey dots represent interlopers and family members, respectively. The vertical lines show border between the real family members and interlopers.

with $a^{*}>3 \sigma\left(a^{*}\right)$ are interlopers. This methodology is illustrated in Fig. 2 for the case of the Klumpkea family. ${ }^{4}$

\subsubsection{The geometric albedos - WISE, AKARI and IRAS}

Another information that could be used to separate $C$ taxonomic class from $S$ taxonomic class, and is available for relatively a large number of asteroids, is the geometric albedo. The vast majority of

\footnotetext{
${ }^{4}$ It should be noted that the Klumpkea family is listed as the Tirela family (FIN 612) in the Nesvorný et al. (2015) classification.
}

these data are obtained by three different infrared surveys. The catalogue of asteroid albedos provided by the Infrared Astronomical Satellite (IRAS) survey was presented by Tedesco et al. (2002), and contains albedos and diameters for 2470 objects. The AKARI survey provides a catalogue with data for 5208 asteroids (Usui et al. 2011; Hasegawa et al. 2013). Finally, most of currently known asteroid albedos are provided by the Wide-field Infrared Survey Explorer (WISE; Mainzer et al. 2011a; Masiero et al. 2011). Based on the data provided by this survey, Masiero et al. (2011) published diameters and albedos for more than 100000 main-belt asteroids.

Aiming here to use these albedos to identify interlopers among potential family members, we need to define strict criteria. In this respect, first a spectral type of considered asteroid family should be determined, and then it should be checked for each potential member if its own albedo is consistent with the spectral type of the family.

In order to determine the dominant taxonomic class of a family, we simply calculate the average albedo $\overline{p_{v}^{*}}$ over all members for which this information is provided by at least one of three available surveys ${ }^{5}$, and check if the obtained value is consistent with one of the spectral types. For this purpose we assume that an albedo of 0.13 is a border between two types, but, similarly as in the case of the SDSS data, we also introduced a safety margin. Therefore, an asteroid family is assigned $C$ type if $\overline{p_{v}^{*}}<0.12$, while family is defined to be of $S$ type if $\overline{p_{v}^{*}}>0.14$.

Most of known families fall in one of two broad spectral types defined above. However, as these two intervals do not overlap, in some cases this causes ambiguity in the determination of family spectral type. Therefore, for families with the average albedo in $[0.12,0.14]$ interval, we used predefined confidence intervals, suitable only for these families. Such an example is the Eos family (see Section 3.4).

Once a spectral type is assigned to a family, all potential members of an inconsistent spectral type are defined as interlopers. As a starting point we used results obtained by DeMeo \& Carry (2013). These authors calculated average albedo $\overline{p_{v}}$, and its corresponding standard deviation $\sigma\left(\overline{p_{v}}\right)$, for different classes of asteroids, using data from three mentioned surveys.

Assuming that albedos of both spectral types $(C$ and $S)$ do not spread more than $3 \sigma$ from their average values, we calculated the corresponding confidence intervals. The average albedo and its corresponding standard deviation for $C$-type objects are $\overline{p_{v}}=0.06$ and $\sigma\left(\overline{p_{v}}\right)=0.01$, respectively; thus, a confidence interval is [0.03, 0.09]. Similarly, $\overline{p_{v}}=0.23$ and $\sigma\left(\overline{p_{v}}\right)=0.02$ for $S$ type, resulting in a confidence interval of $[0.17,0.29]$.

Finally, if a family is of $C$ type, all potential members with $p_{v}-3 \sigma>0.09$ are interlopers. Accordingly, if a family belongs to the $S$ type, interlopers are all potential members having $p_{v}+3 \sigma<0.17$. For asteroid families with pre-defined confidence interval, interlopers are those objects that have $p_{v} \pm 3 \sigma$ outside the corresponding interval.

The above-defined criteria should be good enough even at small sizes, for which Mainzer et al. (2011b) found that the $S$ complex partially overlaps the low albedo $C$ complex.

\footnotetext{
${ }^{5}$ If the albedo is provided by more than one survey, we always give priority to the most recent data.
} 


\subsubsection{The spectroscopic data}

The different taxonomy classifications could also be used to exclude interlopers from asteroid families. Unfortunately these data are available only for a very limited number of asteroids. Still, this information should be more reliable than e.g. SDSS colours. Therefore, we use them in our analysis.

Before we describe in detail how individual interlopers are identified using spectroscopic data, it is important to note that a family spectral class is defined based on the results obtained using colour and albedo information. This is because the number of family members with available spectra is often too small to reliably determine the dominant spectral class of the family.

Therefore, if, following the criteria described above, both colour and albedo data suggest that the family is either $C$ or $S$ class, we proceed with the exclusion of interlopers using spectroscopic data. However, if these two sources of information are not in agreement ${ }^{6}$, we completely omit the spectral data from our analysis.

If applied, this analysis is based on the Tholen (1984), Bus \& Binzel (2002) and DeMeo et al. (2009) classifications. As in the case of albedos, if more classifications provide a spectral type of an asteroid, priority is given to the most recent data.

Tholen (1984) used spectrophotometric results from eight-colour asteroid survey (ECAS) to classify asteroids in different taxonomy groups. The main groups are $C, S$ and $X$. Dark, carbonaceous asteroids are usually part of the $C$ group and they could be divided in a few different classes by comparing slope and maximum wavelength of its spectrum. In this respect, members of the $C$ group are $B, F$, $G$ and $C$ classes. Metallic asteroids are members of the $X$ group that is divided into the $E, M$ and $P$ classes according to the asteroid geometric albedo. Siliceous-stony asteroids are members of the $S$ group. There are some groups similar to $S$, but with slightly different spectrum slope and maximum wavelength, such as $Q, R, V$ and $A$ classes. $D$ and $T$ classes cannot be easily classified in any of three broad groups defined by Tholen (1984). If an asteroid family is mostly composed of asteroids belonging to the $C$ group, then an object is an interloper if according to the Tholen's spectral classification it belongs to one of the following classes: $S, E, M, P, Q, R$, $V, D$ and $T$. Analogously, if a family belongs to the $S$ group, its potential member is an interloper if it belongs to one of the following classes: $C, B, F, G, D, T$ or $E M P$.

Bus \& Binzel (2002) used data from Small Main-Belt Asteroid Spectroscopic Survey, which provided continuous spectrum, but covered smaller wavelength range then the ECAS. This classification divided asteroids in 26 taxonomic classes, with most of the classes being similar to those defined by Tholen (1984), although some Bus \& Binzel classes are separated into subclasses. For example, the $S$ group is separated in $S_{a}, S_{k}, S_{l}, S_{q}, S_{r}$ and $S$ class, and hence, if a family is part of the $C$ group, all asteroids that belong to the $S$ group are identified as interlopers. In this case, we exclude as interlopers asteroids of $V, O, L, D, T, A, R, Q, K$ and $E$ classes. Similarly, if a family belongs to the $S$ group, interlopers are those asteroids that belong to the $C$ group or $B, V, T$ and $D$ classes.

A similar classification logic was applied in the DeMeo et al. (2009) classification, but these authors used also the infrared part of the spectrum. Therefore, their classification is similar to that of Bus and Binzel, with some small modifications that eliminated subclasses $L d, S k$ and $S l$, but added a new $S v$ subclass within $S$ class.

\footnotetext{
${ }^{6}$ This may happen if a family belongs to $X$ type. It is because the SDSS $a^{*}$ colour does not separate $C$ from $X$ type, although these two types are characterized by quite different albedos.
}

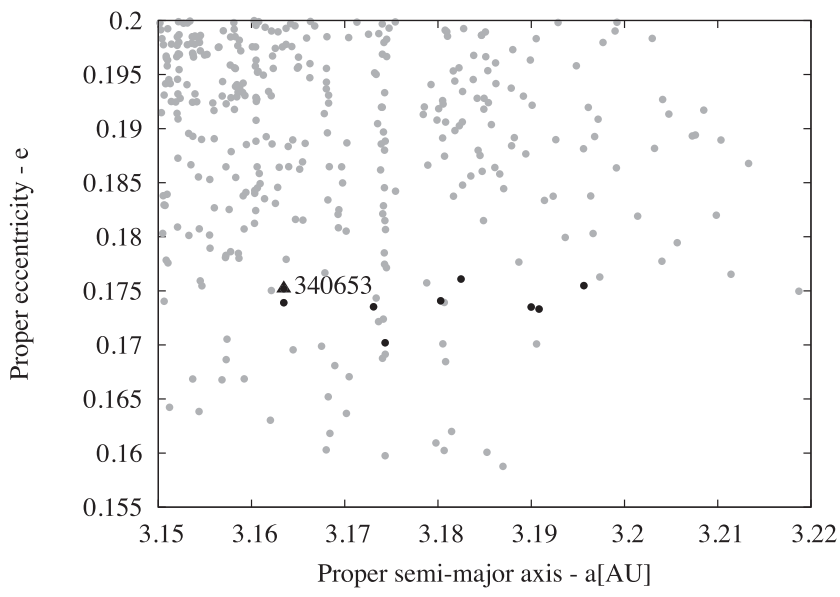

Figure 3. An example of the chaining effect in the Klumpkea family. Black triangle shows location of asteroid (340653) that is identified as an interloper, while black circles represent the eighth additional interlopers, linked to the family due to the chaining through the asteroid (340653). Grey dots mark other family members.

\subsection{Step 3 and step 4}

\subsubsection{Excluding interlopers}

In the third step, asteroids identified as interlopers due to their reflectance characteristics are excluded from the initial catalogue of proper elements. This produces a modified catalogue that is free of known interlopers in the corresponding family. Therefore, the interlopers are excluded from the catalogue of proper elements, rather than from the list of family members, as it is usually done.

In the fourth step the HCM is applied again to the modified catalogue of proper elements, produced in the third step. This is the main difference from the methods that have already been used for determining interlopers in asteroid families. The point of this step is to reduce the chaining effect in the HCM. By removing interlopers from the catalogue, we have also removed objects linked to the family through some of these interlopers. An example of the efficiency of this methodology in the Klumpkea family is shown in Fig. 3. Asteroid (340653) is identified as an interloper based on the albedo data. Once it is removed from the input catalogue, the eighth additional objects are removed.

\subsubsection{The final membership}

Finally, in the fourth step, the family members are re-identified using the modified catalogue of proper elements, produced in the third step by removing interlopers identified based on colour, albedo and spectral data. For a purpose of comparison, we run the HCM on the modified catalogue using again the cut-off distance adopted in the first step. This allows us to estimate the fraction of the removed interlopers within each family.

On the other hand, as discussed above, the cut-off used in the first step is too high to obtain the nominal membership of an asteroid family. A large cut-off value may easily associate many background asteroids to a family. This may even create bridge between different families resulting in a wrong membership. Therefore, the final membership of each family should be obtained using the cut-off that describes a family the best on the modified catalogue of proper elements. Hence, the determination of a nominal cut-off is a very important step in this analysis, and should be carefully performed. 


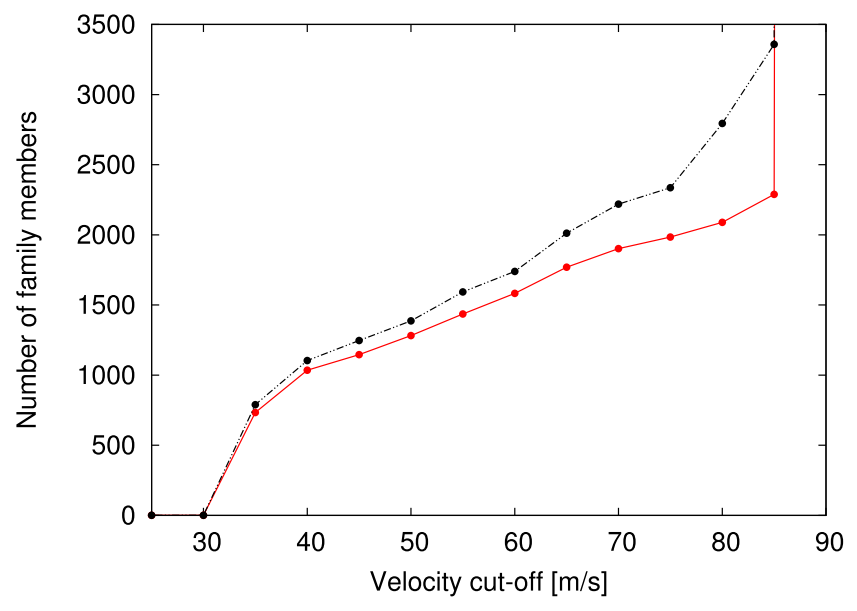

Figure 4. Number of asteroids linked to the Klumpkea family. The dashed and solid lines represent data obtained using the initial and modified catalogues, respectively.

The method used here to select the nominal cut-off value is as follows. First, it is checked how the number of family members changes as a function of cut-off values (Fig. 4). Then, in order to estimate a nominal cut-off we search for a plateau, i.e. an interval of cut-off values where a number of family members are almost constant. As a general rule, if such a plateau is well defined, the nominal cut-off is adopted to be around the centre of the plateau. Still, in some cases the plateau practically does not exist, and a detailed analysis of corresponding family should be performed in order to determine its nominal cut-off value. The latter cases are discussed below, in Section 3.4.

\section{RESULTS}

In this section we demonstrate how the above-described method works, and present the most important results. For this purpose we selected 17 large families from the classification of Milani et al.
Table 2. Average colour and albedo characteristics of analysed families. The columns are (1) family name (Family), (2) average SDSS $a^{*}$ colour $\left(a_{\mathrm{m}}^{*}\right)$, (3) standard deviation of average $a^{*}$ colour $\left(\sigma_{\mathrm{m}}\left(a^{*}\right)\right)$, (4) average albedo $\left(p_{v}^{*}\right),(5)$ standard deviation of average albedo $\left(\sigma\left(p_{v}^{*}\right)\right)$ and (6) determined family type (Type)

\begin{tabular}{lrcccc}
\hline Family & \multicolumn{1}{c}{$a_{\mathrm{m}}^{*}$} & $\sigma_{\mathrm{m}}\left(a^{*}\right)$ & $p_{v}^{*}$ & $\sigma\left(p_{v}^{*}\right)$ & Type \\
\hline (5) Astraea & 0.09 & 0.03 & 0.19 & 0.06 & $S$ \\
(10) Hygiea & -0.10 & 0.03 & 0.07 & 0.02 & $C$ \\
(15) Eunomia & 0.08 & 0.03 & 0.20 & 0.05 & $S$ \\
(20) Massalia & 0.07 & 0.04 & 0.24 & 0.09 & $S$ \\
(24) Themis & -0.11 & 0.03 & 0.07 & 0.02 & $C$ \\
(135) Hertha & 0.08 & 0.03 & 0.17 & 0.06 & $S$ \\
(145) Adeona & -0.09 & 0.04 & 0.07 & 0.02 & $C$ \\
(158) Koronis & 0.09 & 0.03 & 0.23 & 0.07 & $S$ \\
(170) Maria & 0.11 & 0.03 & 0.25 & 0.08 & $S$ \\
(221) Eos & 0.03 & 0.03 & 0.13 & 0.04 & $S$ \\
(490) Veritas & -0.07 & 0.03 & 0.07 & 0.02 & $C$ \\
(668) Dora & -0.11 & 0.03 & 0.06 & 0.02 & $C$ \\
(847) Agnia & 0.07 & 0.03 & 0.19 & 0.07 & $S$ \\
(1040) Klumpkea & 0.11 & 0.03 & 0.14 & 0.04 & $S$ \\
(1726) Hoffmeister & -0.09 & 0.03 & 0.05 & 0.02 & $C$ \\
(2076) Levin & 0.04 & 0.03 & 0.21 & 0.07 & $S$ \\
\hline
\end{tabular}

(2014), each of them with more than 1000 potential members. These families are statistically reliable, but at the same time are also expected to have a large number of interlopers.

Before discussing these results, let us to recall that they are obtained using unrealistically high-velocity distance cut-off values $\left(d_{\text {cut }}\right)$. The purpose here is only to show how the method works, and in particular to highlight the importance of our step 4. Realistic results, obtained using the most appropriate values of $d_{\text {cut }}$, are given below in Section 3.4.

The results for the 17 analysed families are summarized in Tables 1 and 2. The total fraction of interlopers found among potential family members vary from below 1 per cent for families well separated from the background population (e.g. the Hoffmeis-

Table 1. Summary of results on excluding interlopers from selected families. The columns are (1) family name (Family), (2) adopted cut-off value ( $\left.d_{\text {cut }}\right)$, (3) number of asteroids as family members identified using cut-off values adopted in step 1 (\# STEP1), (4) number of interlopers identified using SDSS colours (\# SDSS), (5) number of interlopers identified using albedos (\# ALBEDO), (6) number of interlopers identified using available taxonomic classifications (\# TAX.), (7) total number of interlopers based on the reflectance spectra (\# STEP2), (8) number of asteroids excluded due to the chaining effect (Chaining), (9) number of asteroids identified as family members using adopted $d_{\text {cut }}$ and modified input catalogue (\# STEP4) and (10) a fraction of excluded asteroids (per cent).

\begin{tabular}{|c|c|c|c|c|c|c|c|c|c|}
\hline Family & $d_{\text {cut }}$ & \#STEP1 & \#SDSS & \#ALBEDO & \#TAX. & \#STEP2 & Chaining & \#STEP4 & $\%$ \\
\hline (10) Hygiea & 70 & 5904 & 24 & 15 & 3 & 38 & 86 & 5780 & 2.1 \\
\hline (20) Massalia & 30 & 4663 & 7 & 8 & 0 & 13 & 2 & 4648 & 0.3 \\
\hline (24) Themis & 80 & 5499 & 20 & 39 & 0 & 59 & 31 & 5409 & 1.6 \\
\hline (93) Minerva & 75 & 7015 & 352 & 845 & 39 & 1057 & - & - & - \\
\hline (158) Koronis & 65 & 7743 & 28 & 81 & 1 & 101 & 38 & 7604 & 1.8 \\
\hline (170) Maria & 60 & 2939 & 20 & 28 & 0 & 45 & 44 & 2850 & 3.0 \\
\hline (221) Eos & 70 & 24155 & 555 & 1706 & 39 & 2089 & 757 & 21309 & 11.8 \\
\hline (490) Veritas & 30 & 1295 & 6 & 7 & 0 & 13 & 0 & 1282 & 1.0 \\
\hline (668) Dora & 60 & 1401 & 9 & 3 & 1 & 13 & 0 & 1388 & 0.9 \\
\hline (847) Agnia & 45 & 3054 & 14 & 52 & 0 & 61 & 84 & 2909 & 4.7 \\
\hline
\end{tabular}



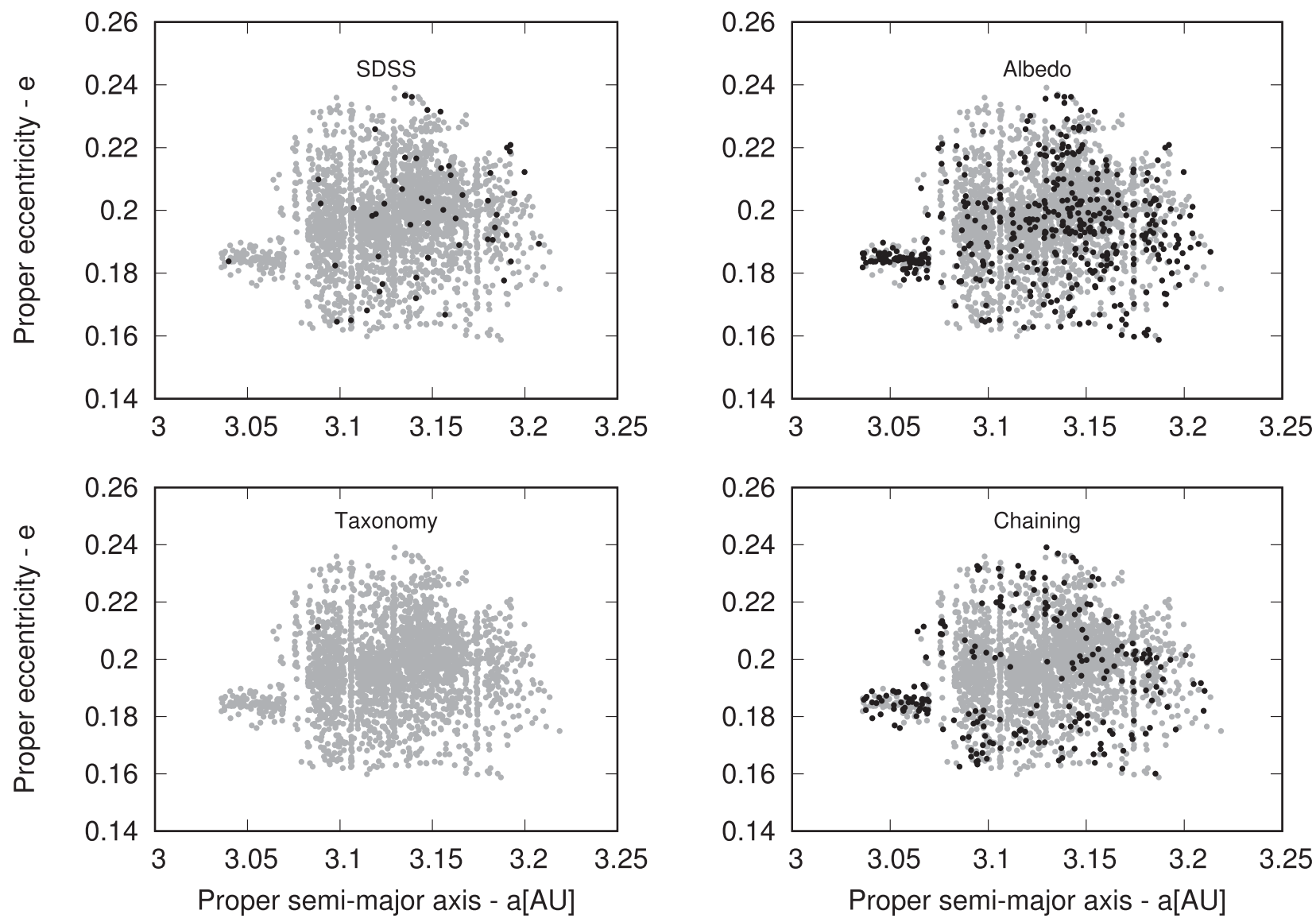

Figure 5. Distribution of interlopers within the Klumpkea family in the $a-e$ plane. Each panel shows interlopers identified using different information, as written in the panels. Black and grey circles mark interlopers and all asteroids linked to the family, respectively.

ter family) to more than 20 per cent for cases where an additional family may be present, as for example in the case of the Klumpkea family. Despite these differences, the results are generally in agreement with the expected fraction of interlopers estimated by Migliorini et al. (1995).

To better appreciate the importance of step 4, which is the main improvement of our approach with respect to previous works, one should compare the numbers shown in columns 7 and 8 of Table 1. The seventh column gives the total number of interlopers identified using available physical and spectral data (\#STEP2), while the eighth column provides the number of asteroids linked to the family through some of the identified interlopers (Chaining).

The numbers in these two columns are often comparable, and in some cases the number of interlopers removed due to chaining effect is even larger than the number of interlopers identified based on the physical and spectral data. The only exceptions are families with a very small number of interlopers (e.g. Veritas and Hoffmeister), and cases where two or more families overlap (e.g. Eunomia family). Nevertheless, usage of step 4 seems to be important and fully justified.

In order to further demonstrate how the method works we selected two families as the case studies, namely the (1040) Klumpkea and (15) Eunomia families. The Klumpkea family was selected due to its location within the main belt. It is situated in the outer part of the main belt, where most of the asteroids belong to $C$ spectral type, while Klumpkea belongs to $S$ type. Therefore, we expect to find a relatively large fraction of the interlopers among members of the Klumpkea family. The Eunomia family is a large group in the middle main belt, and it is an interesting example because there may be another family buried inside the Eunomia family.

\subsection{The case study no. 1: Klumpkea family}

Analysing the outputs of the HCM applied to the initial catalogue for different cut-off values, it could be noted that for cut-off below $55 \mathrm{~m} \mathrm{~s}^{-1}$, Klumpkea family members are situated between the $11 / 5$ and 21/10 mean motion resonances with Jupiter. Starting from $55 \mathrm{~m} \mathrm{~s}^{-1}$ family members are crossing the 21/10 resonance, while at $80 \mathrm{~m} \mathrm{~s}^{-1}$ family members cross also the $11 / 5$ resonance. At the cut-off velocity of $90 \mathrm{~m} \mathrm{~s}^{-1}$, the family merges with the background population of asteroids. Therefore, the cut-off value of $80 \mathrm{~m} \mathrm{~s}^{-1}$ is adopted for step 1, yielding the initial family membership that contains 2794 asteroids (see Fig. 4). These potential members of Klumpkea family have an average albedo $\overline{p_{v}}=0.142 \pm 0.044$, and an average SDSS colour $\overline{a^{*}}=0.112 \pm 0.035$. Hence, according to the criteria explained in Section 2.2 the family belongs to the $S$ type.

In the second step, among the initial family members 452 interlopers are detected (see Table 1). Fig. 5 shows the distribution of these interlopers in different planes. It should be noted that most of the potential members located at $a<3.075$ au are likely interlopers.

After removing the interlopers from the initial catalogue, and applying the HCM to the modified catalogue ${ }^{7}, 2115$ asteroids were identified as family members. Therefore, there are 679 members less

\footnotetext{
${ }^{7}$ Using the same $d_{\text {cut }}$ as in the first step.
} 

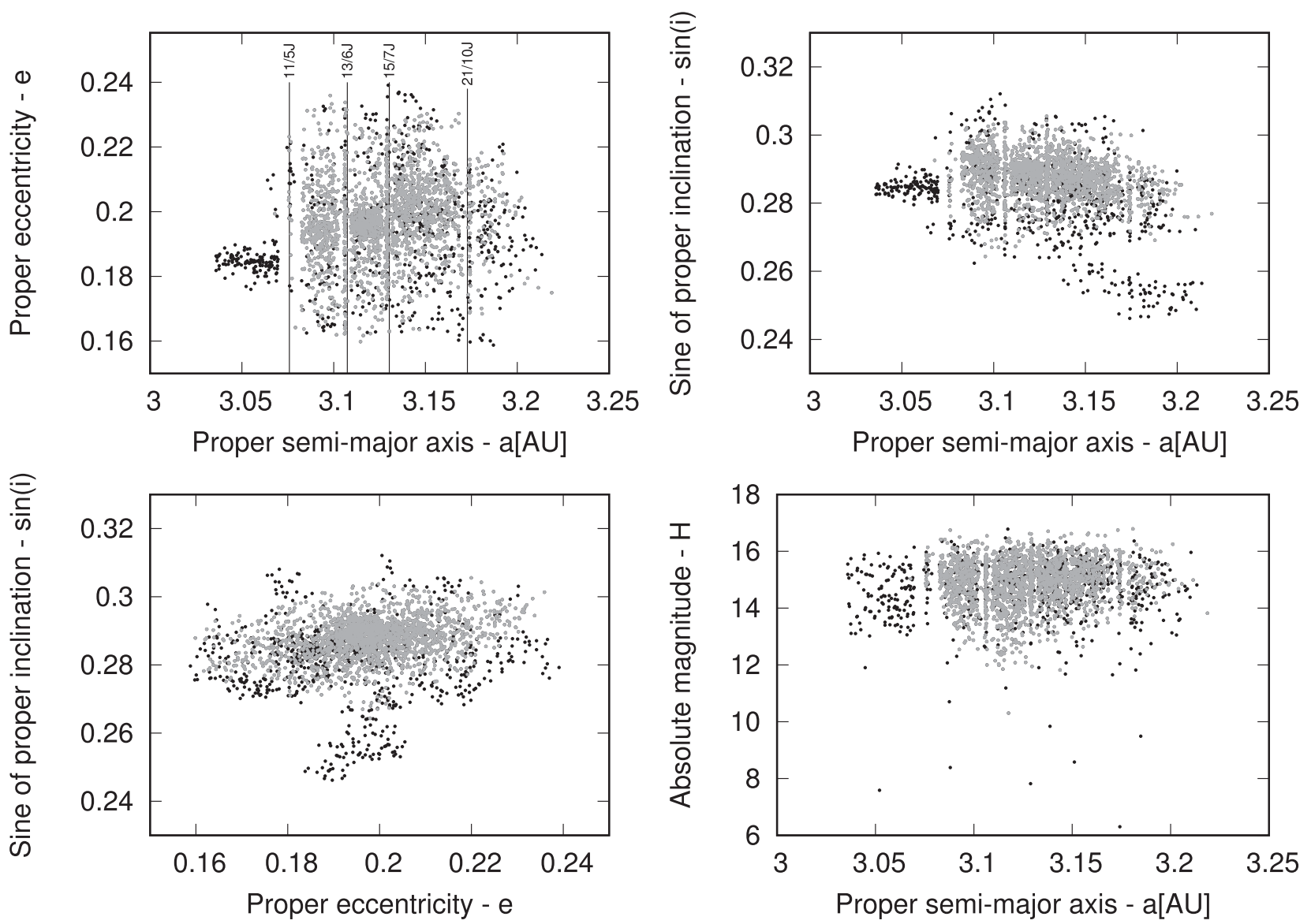

Figure 6. The final membership (grey) and all identified interlopers (black) of the Klumpkea family, shown in the space of proper elements and absolute magnitude. The vertical lines in the top-left panel denote locations of the most important mean-motion resonances that cross the family.

than in the initial step, and among these 227 asteroids are removed in step 4 due to the chaining effect.

In order to determine the nominal cut-off for the Klumpkea family, we study again the HCM results, but this time obtained on the modified catalogue. The situation seems to be different with respect to the results obtained before removal of interlopers. The most important new feature is that family members do not cross the $11 / 5$ resonance, for cut-off values below $85 \mathrm{~m} \mathrm{~s}^{-1}$ when the family merges with the local background population (Fig. 6). These results are consistent with two recent classification performed by Milani et al. (2014) and Nesvorný et al. (2015), where the authors found that at the inner side of the $11 / 5$ resonance there is another family, namely that of (96) Aegle.

Moreover, a low inclination part of the initial family is completely removed (see upper-right panel of Fig. 6). Finally, it is interesting to note that in the $a-H$ plane (lower-right panel of Fig. 6) most of the objects located below the $\mathrm{V}$ shape are identified as interlopers, and the removal of these asteroids improve the visibility of the family V shape. ${ }^{8}$

\footnotetext{
${ }^{8}$ The so-called V shape is a characteristic shape of the real collisional families when projected on the semimajor axis versus absolute magnitude (or inverse diameter) plane. It is mainly the result of the action of Yarkovsky thermal force which disperses more quickly smaller objects (Vokrouhlický et al. 2015). The shape structure is mostly used to estimate the age of a family (Vokrouhlický et al. 2006; Milani et al. 2014; Spoto, Milani \& Knežević
}

For the nominal cut-off value of the Klumpkea family we adopt $d_{\text {nom }}=60 \mathrm{~m} \mathrm{~s}^{-1}$, which corresponds to the removal of 9.8 per cent of asteroids initially linked to the family. About 18 per cent of interlopers are removed in the fourth step of our procedure, which is introduced to reduce the chaining effect.

\subsection{The case study no. 2: Eunomia family}

To get the initial list of family members, we start from the cutoff value of $5 \mathrm{~m} \mathrm{~s}^{-1}$ and increase it until the family merges with background asteroids (Fig. 7). Based on the defined criteria we adopt a cut-off value of $60 \mathrm{~m} \mathrm{~s}^{-1}$, resulting in 11889 potential members.

The above-defined Eunomia family has an average albedo $\overline{p_{v}}=$ $0.199 \pm 0.056$, and average SDSS colour $\overline{a^{*}}=0.084 \pm 0.030$, suggesting that this family belongs to the $S$ type.

In the preliminary list of members we found 1595 interlopers (see Table 1 for more details), whose distributions are shown in Fig. 8. Applying the HCM with the same $d_{\text {cut }}$ as in the first step to the modified catalogue, 9978 asteroids are identified as the family members (Figs 8 and 9). Therefore, there are 1911 (about 16 per cent) asteroids linked to the family less than in the initial step, with 316 of them rejected in step 4.

2015), but could also be used to search or confirm existence of asteroid families (Walsh et al. 2013; Bolin et al. 2017). 


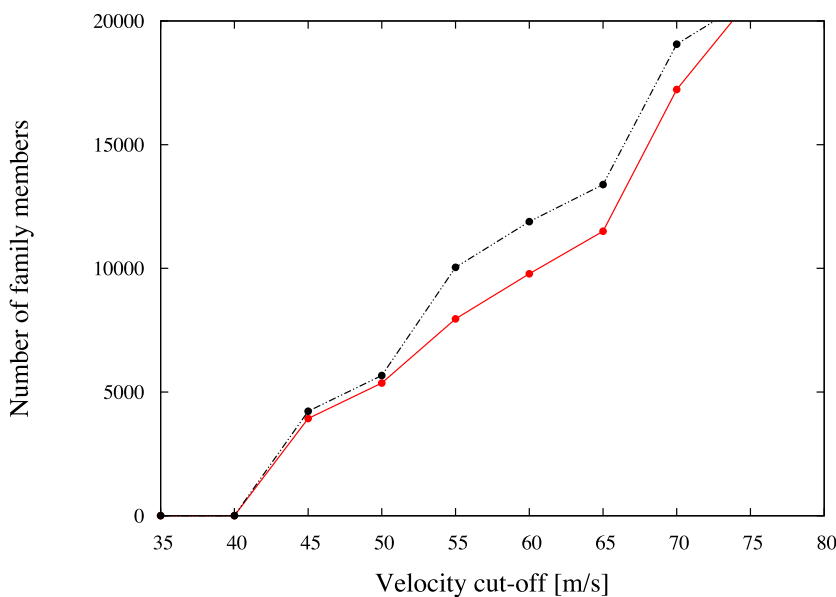

Figure 7. Number of asteroids linked to the Eunomia family. The dashed and solid lines represent membership obtained using the initial and modified catalogues, respectively.

A cut-off value of $55 \mathrm{~m} \mathrm{~s}^{-1}$ is adopted as a nominal to define the family, resulting in removal of almost 20 per cent of asteroids linked to the family as interlopers. Migliorini et al. (1995) analysed the Eunomia family and found that a large fraction of interlopers $\sim 20$ per cent should indeed be expected in this family, in agreement with the results obtained in our work. A possible explanation for such a large fraction of interlopers may be the presence of another asteroid family. Additional argument in favour of this hypothesis is
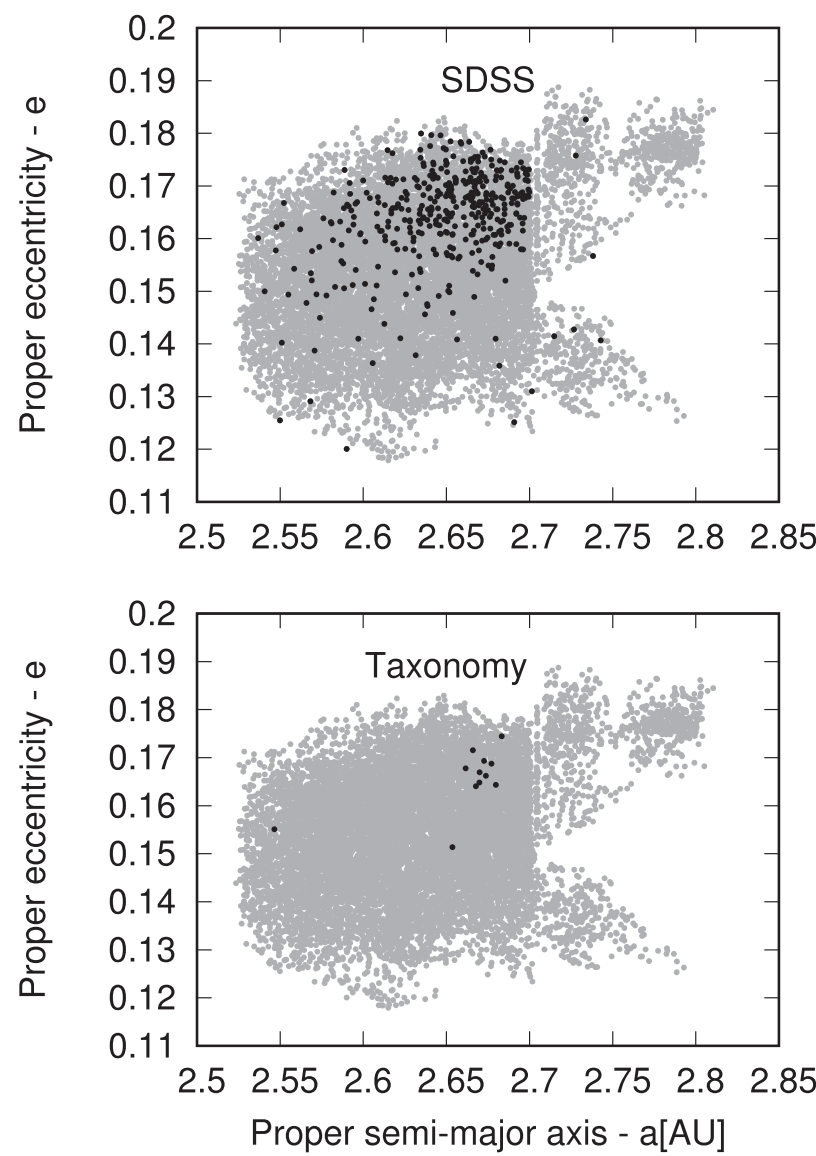

concentration of interlopers in specific part of the Eunomia family (see Fig. 8). Examining the available data we found that $C$-type Adeona should be this overlapping family, as its location corresponds to the groupings of interlopers visible within the borders of the Eunomia family.

Examining the V-shaped structure of the Eunomia family shown in Fig. 9, we noted that it is very well defined, and practically all objects positioned outside the $\mathrm{V}$ shape are identified as interlopers. Moreover, even the interlopers form a visible V-shaped structure, although cut at outer side in terms of the semimajor axis. This is an additional evidence that most of the interlopers belong to another collisional family, i.e. to the Adeona family.

The results of this case study illustrate the efficiency of the method developed here, when dealing with two overlapping families of different spectral types.

\subsection{Special cases}

The special cases presented in this subsection refer to groups composed of two or more overlapping collisional families. Aiming to keep our approach fully automatic and relatively simple, obviously we do not expect to completely solve these complex cases. Moreover, as we are distinguishing here only between the $C$ and $S$ taxonomic complexes, we could not resolve more than two overlapping families. Therefore, the main purpose of this part is to demonstrate how our method works in these cases, rather than to provide a full explanation for them.
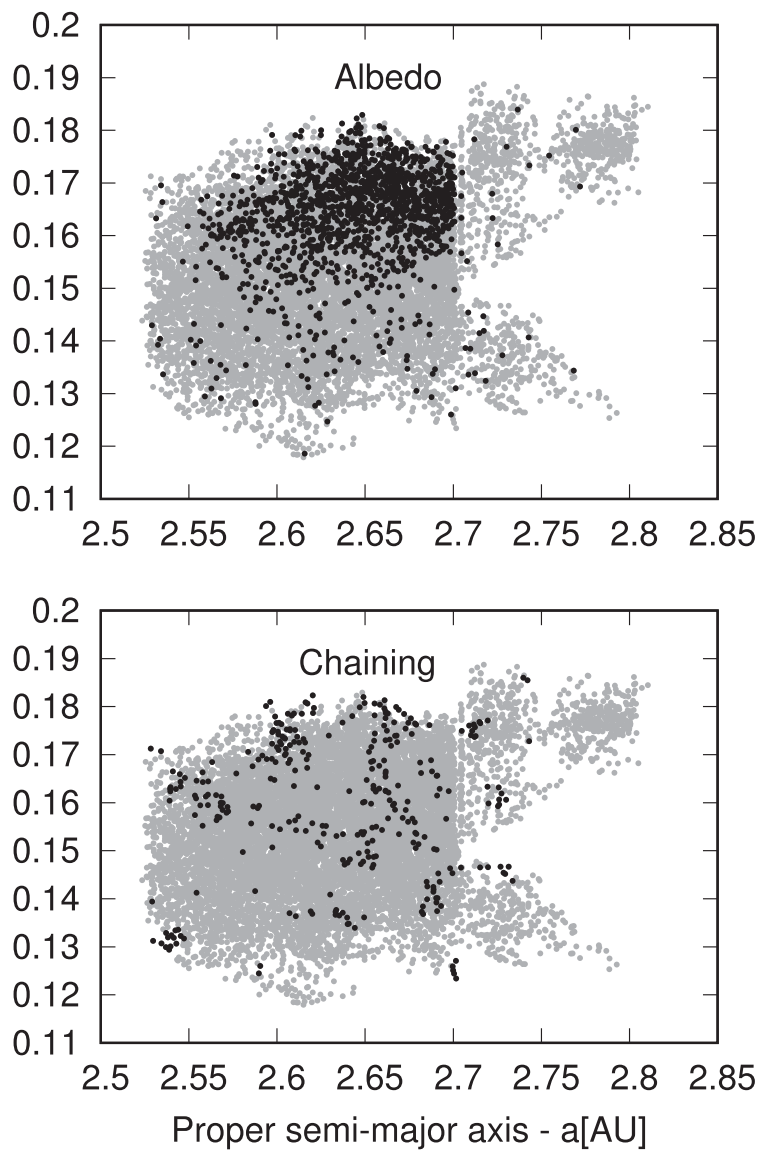

Figure 8. Distribution of interlopers within Eunomia family in the $a-e$ plane. The grey points represent family members, while the black points mark interlopers. 

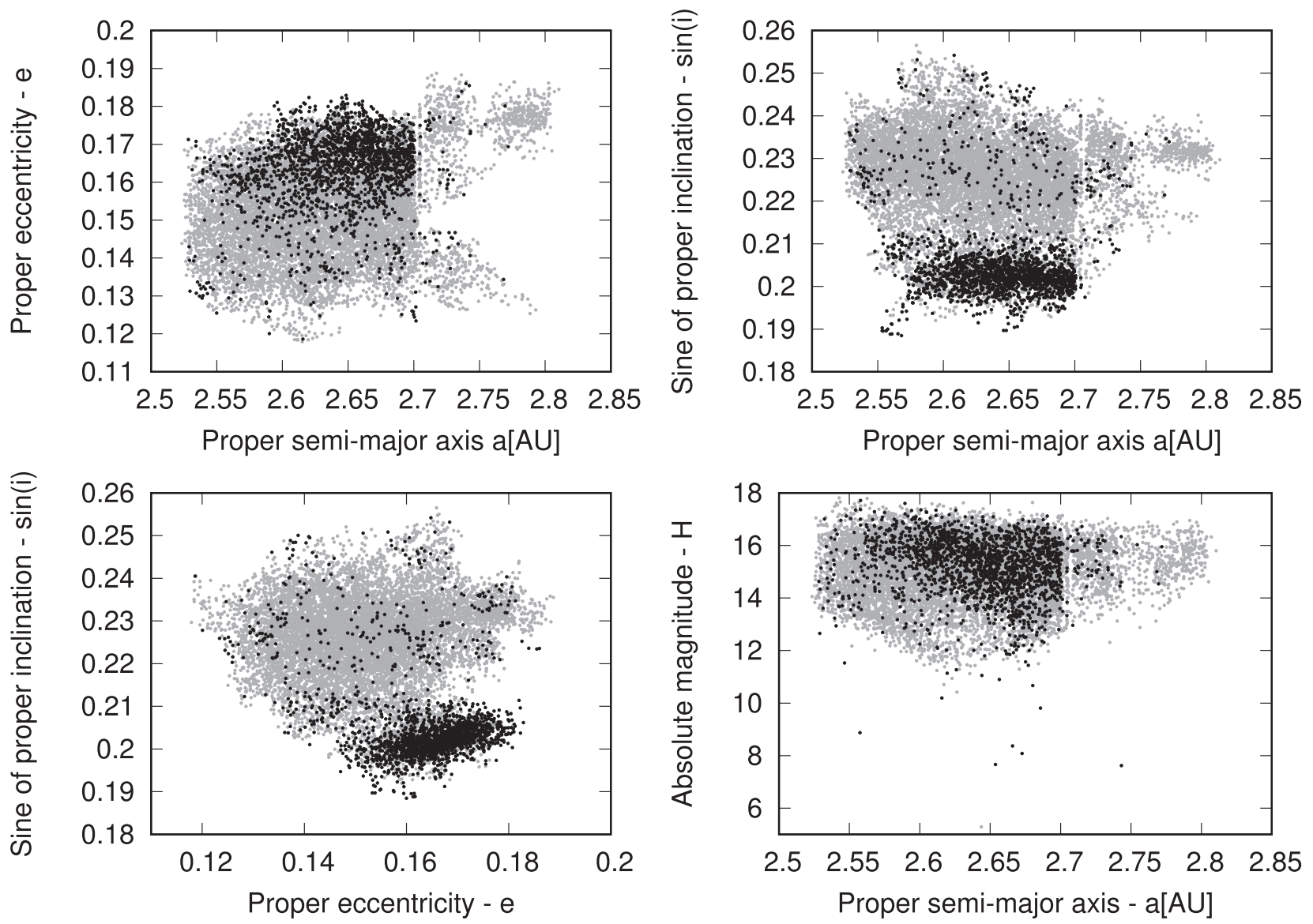

Figure 9. The final membership (grey) and all identified interlopers (black) of the Eunomia family.

\subsubsection{Minerva clan}

The asteroids from the regions around (1) Ceres are strongly perturbed by the nearby secular resonances with Ceres (Novaković et al. 2015; Tsirvoulis \& Novaković 2016). The existence of these resonances complicates any attempt to identify asteroid families.

Asteroid Minerva was first considered as a part of Ceres dynamical family (Zappalà et al. 1995). The latter works also recognized this group, but its largest associated object has changed. It was proposed that asteroid Ceres is not a member of this group, ${ }^{9}$ making (93) Minerva the largest member, and consequently the group is named Minerva family. However, later on it was realized that this family mainly consists of $S$-type asteroids, indicating that $C$-type Minerva asteroid is actually an interloper. Thus, the family name has changed once again to Gefion family.

As Milani et al. (2014) identified the so-called dynamical families, ${ }^{10}$ their classification includes the Minerva family. Therefore, we applied our analysis to this group, but note that asteroid (93) Minerva may not be a parent body of this group.

In the initial step, applied using cut-off of $75 \mathrm{~m} \mathrm{~s}^{-1}$ and asteroid (93) Minerva as a central object, we identified a group of $7015 \mathrm{mem}-$ bers. As this group includes more than one family (see discussion

\footnotetext{
${ }^{9}$ For a discussion on the missing Ceres family, see Milani et al. (2014), Rivkin, Asphaug \& Bottke (2014) and Carruba et al. (2016).

${ }^{10}$ Dynamical families are groupings of asteroids identified using purely dynamical characteristics, i.e. proper orbital elements. Therefore, they may not necessarily represent collisional families.
}

below), we refer to it as Minerva clan (Cellino \& Zappala 1993). In the second step of our procedure applied to the Minerva clan, we calculated the average colour: $\overline{a_{*}}=0.044 \pm 0.032$ and albedo: $\overline{p_{v}}=0.137 \pm 0.04$. Both these values are somewhere in between the typical values for the $S$ type and the $C$ type. While according to our criteria colour data suggest the group should be marginally classified as the $S$ type, the albedo value falls exactly between the values used to discriminate between the $C$ type and the $S$ type. This is an indication that the Minerva clan includes asteroids of different composition and possibly more than one single family. This is further supported by the fact that the Minerva clan, as identified here, includes asteroids (1) Ceres, (668) Dora and (1272) Gefion.

Due to existence of asteroids of different taxonomic types, we continue in two directions; that is, we first perform analysis assuming that the Minerva clan is $S$ type, but then repeated this step assuming it is $C$ type. In the first case, we found 1497 interlopers, objects not compatible with $S$-type taxonomy. Among them there are asteroids (1), (93) and (668). After removing the interlopers from the initial catalogue, we obtained the modified catalogue of proper elements. Because asteroid (93) Minerva is not present in the new catalogue, we applied the HCM analysis using (1272) Gefion as a central body. For nominal cut-off value in this case we adopted $d_{\text {nom }}=50 \mathrm{~m} \mathrm{~s}^{-1}$, and obtained well-defined Gefion family with 2306 members.

For the second case we assume Minerva clan is $C$ type and, in step 2, identified 985 asteroids as interlopers, including (1272) Gefion. Again, we exclude them from the initial catalogue, and apply the HCM using nominal cut-off of $65 \mathrm{~m} \mathrm{~s}^{-1}$ and asteroid Minerva as 


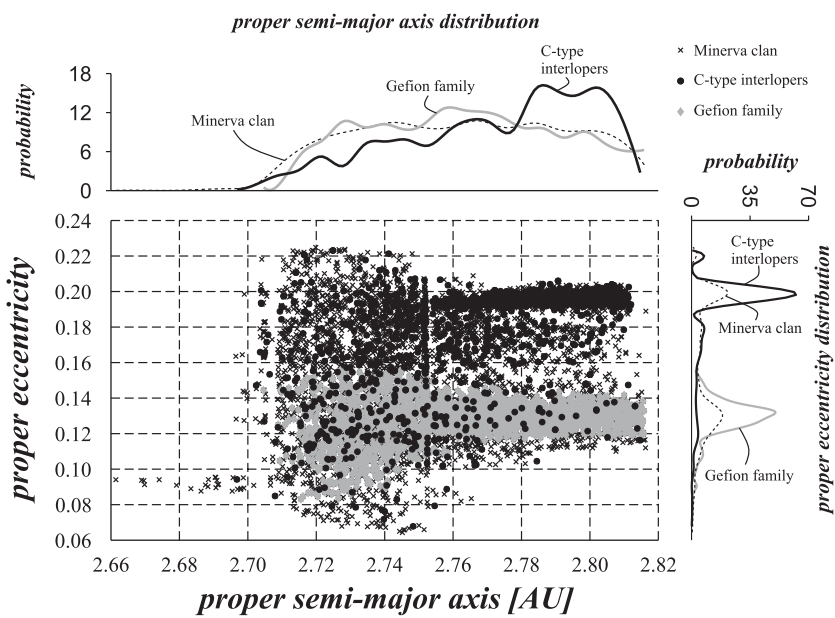

Figure 10. The asteroids linked to the Minerva clan, along with its $C$-type interlopers and the Gefion family. The large concentration of dark interlopers visible in the upper-right part of the panel corresponds to the Dora family. Note that apart from this grouping, the distribution of $C$-type interlopers is almost uniform, suggesting that the dark Minerva family actually does not exist. See the text for additional explanation. In the top and right small panel, probability density functions of the proper elements are given. These functions are obtained by interpolation of the normalized histograms.

a central body. To this group of 3184 asteroids we refer here as a Minerva group. Our results show that difference between the initial and the final Minerva group is 1356 asteroids. This large fraction of interlopers could be explained by the presence of Gefion family members, which we identify as a separate family composed of $S$ type asteroids.

As shown in Fig. 10, the Minerva group and Gefion family, as defined here, partly overlap in the proper elements space. In order to better understand the real nature of the Minerva group, we analysed the distribution of the family members in orbital space. We note that the largest concentration in the Minerva group coincides with the location of the Gefion family. On the other hand the distribution of the dark asteroids in this region is roughly uniform (Fig. 10), except at the location of the Dora family that was included in the membership of the Minerva group. Based on this evidence, we concluded that asteroids belonging to the Minerva group that coincide with the location of the Gefion family are actually the members of the latter family for which albedo and/or colour data are not available.

The above-mentioned facts imply that the Minerva dynamical family is not a collisional family, while nearby there is one bright (Gefion) and one dark (Dora) family. Still, caution is needed before any definite conclusion is derived about the families in this region. The secular resonances with Ceres complicate any attempt to identify asteroid families, and instead of the HCM, other techniques should be used to search for potential families, as explained in Carruba et al. (2016).

\subsubsection{Hertha/Nysa-Polana clan}

The Hertha family is one of the largest families that is considered in this paper. Other paper usually referred to it as Nysa-Polana complex of asteroids. This complex was first proposed as a single family by Brouwer (1951), but later data showed that there are two or more overlapping collisional families (Cellino et al. 2001; Walsh et al. 2013; Dykhuis \& Greenberg 2015). As this group is known to contain multiple families, we will refer to it as Hertha clan.

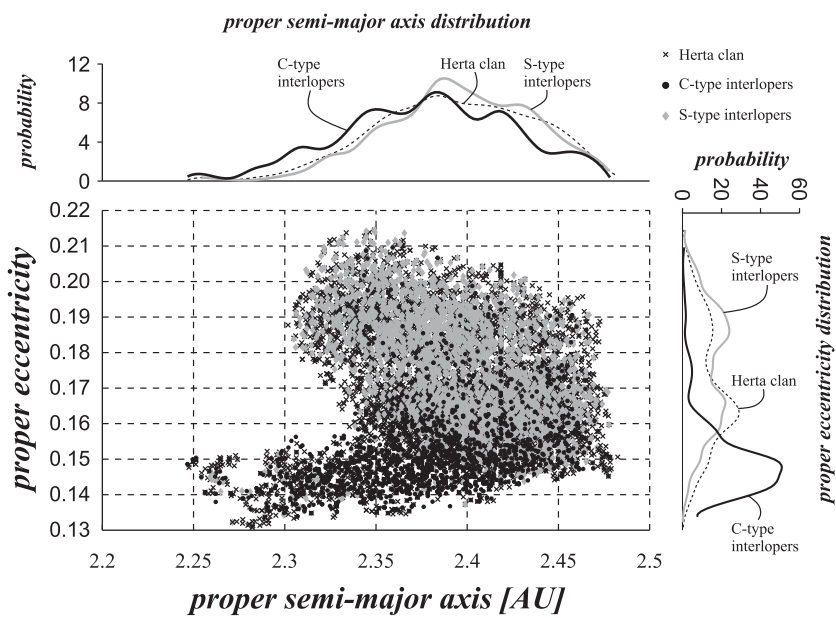

Figure 11. The $C$ - and $S$-type interlopers within the Hertha clan. The distribution of these interlopers is clearly not random, but shows concentration around different values of proper eccentricity, indicating a presence of more than a single collisional family. In the top and right small panel, probability density functions of the proper elements are given. A small offset in terms of proper semimajor, between the main concentrations of $C$ - and $S$-type interlopers, is notable (top panel), but these two groups are obviously much better separated in terms of proper eccentricity (see right-hand panel).

Following our approach, in the first step by using the cut-off value of $45 \mathrm{~m} \mathrm{~s}^{-1} 22851$ asteroids are identified as members of the Hertha clan (Fig. 11). According to the described criteria, the Hertha clan should be classified as $S$ type. Interestingly, the average colour and albedo do not show any indication that there may exist a family of different spectral types (see Table 2). This may be because among the objects belonging to the Hertha clan, the $S$-type asteroids with available spectral data are more numerous than the $C$-type ones.

Being similar to the Minerva clan, we employed the same approach here in order to define families within the Hertha clan, i.e. we did not use only the spectral type of the clan derived in the second step, but run the procedure twice, once assuming the family is $S$ type, and once assuming it is $C$ type.

In the first run we identified $1603 C$-type asteroids as interlopers, including asteroid (142) Polana. After excluding $C$-type interlopers from the initial catalogue of proper elements, the HCM analysis is performed using modified catalogue. For a nominal cut-off of $35 \mathrm{~m} \mathrm{~s}^{-1}, 9815$ asteroids are associated with the family, with asteroid (135) Hertha as it is the lowest numbered member. Therefore, after excluding $C$-type asteroids, the Hertha family is obtained. ${ }^{11}$ Asteroid (44) Nysa is included in the Hertha family, but at a larger cut-off value of $45 \mathrm{~m} \mathrm{~s}^{-1}$. Interestingly, Dykhuis \& Greenberg (2015) proposed that the Hertha family consists of two sub-families, namely Hertha 1 and Hertha 2; however, our methodology is not able to provide any evidence in this respect.

In the second run, $2537 S$-type asteroids are identified as interlopers, and among these there are (20) Massalia, (44) Nysa and (135) Hertha asteroids. Therefore, in order to obtain a $C$-type

\footnotetext{
11 The second largest asteroid in the Hertha family identified in this work is (878) Mildred. Cellino et al. (2001) excluded asteroid (135) Hertha from its namesake family due to difference in spectrum of (135) Hertha and the rest of family members. Therefore, these authors used asteroid (878) as the parent body of the family that they called Mildred family. As our conservative criteria did not exclude asteroid (135) Hertha from the family membership, we still called it the Hertha family.
} 
family within the Hertha clan, these $S$-type asteroids are excluded as interlopers. After doing this, asteroids (135) Hertha and (44) Nysa no longer belong to the Hertha clan. For this reason, the next largest asteroid (142) Polana is used as a central body in order to define a new family. The HCM at the cut-off velocity of $45 \mathrm{~m} \mathrm{~s}^{-1}$ listed 11522 asteroids as members of the Polana family. This group seems to be even more complex, and potentially contains more than a single collisional family. Other works refer to this part of the Hertha clan as the Eulalia family (Walsh et al. 2013) or even three separate families (Dykhuis \& Greenberg 2015).

Distributions of $C$ - and $S$-type interlopers within the Hertha clan are shown in Fig. 11. The interlopers of the two spectral types are concentrated in two separate groups, revealing the presence of at least two collisional families. In Fig. 11, it could be seen that $C$-type interlopers are mostly located at smaller proper eccentricities, while the $S$-type interlopers groups are at somewhat larger values. ${ }^{12}$

In conclusion, we successfully distinguished two separate groups within the Hertha clan, namely the Hertha and Polana families. A further distinction among additional overlapping sub-families requires detailed study of these groups, and probably a somewhat different methodology. This is beyond the scope of our automatic approach that is designed to be as simple as possible.

\subsubsection{The potential Levin family}

The existence of an asteroid family around (2076) Levin was first proposed by Milani et al. (2014). The Levin family is located in the inner part of the main belt, where both large number density of asteroids and complex dynamical environment make any attempt to identify families more difficult. Moreover, it seems that some families share this part of the orbital elements space, by overlapping each other.

Applying our step 1, we found that the concentration of asteroids around (2076) Levin merges with background population at $55 \mathrm{~m} \mathrm{~s}^{-1}$. Thus, our selection criteria yields to cut-off value of $45 \mathrm{~m} \mathrm{~s}^{-1}$. It is important to note here that the Levin family defined at $45 \mathrm{~m} \mathrm{~s}^{-1}$ also includes the (298) Baptistina family, although the latter was treated as a separate group in Milani et al. (2014).

Actually, the membership of the Baptistina family, as defined at PDS ${ }^{13}$ by Nesvorný et al. (2015), exactly matches the membership of our Levin family obtained at $45 \mathrm{~m} \mathrm{~s}^{-1}$. A similar definition of the Baptistina family was also proposed by Masiero et al. (2012). In this respect, there is some disagreement whether the Levin and Baptistina are different families, or they belong to a single family. Therefore, it would be interesting to see how removal of potential interlopers would influence the situation.

Our search for interlopers among members of the Levin family, performed within steps 2 and 3, did not result in two separated families at $45 \mathrm{~m} \mathrm{~s}^{-1}$. This might be an indication that the Levin and Baptistina belong to a single collisional family. However, the large number density of asteroids in this region also complicates a reliable identification of interlopers, thus caution is needed. Still, a close inspection of the family V shape caused by the Yarkovsky effect suggests that both groups may belong to the same family (see Fig. 12).

\footnotetext{
${ }^{12}$ It should be noted that although we are calling these objects interlopers, they are likely members of one of these two overlapping families. Hence, the term interlopers is used to denote that objects for which we know that they do not belong to the $C$ - or $S$-type family.

${ }^{13} \mathrm{http}: / /$ sbn.psi.edu/pds/resource/nesvornyfam.html
}

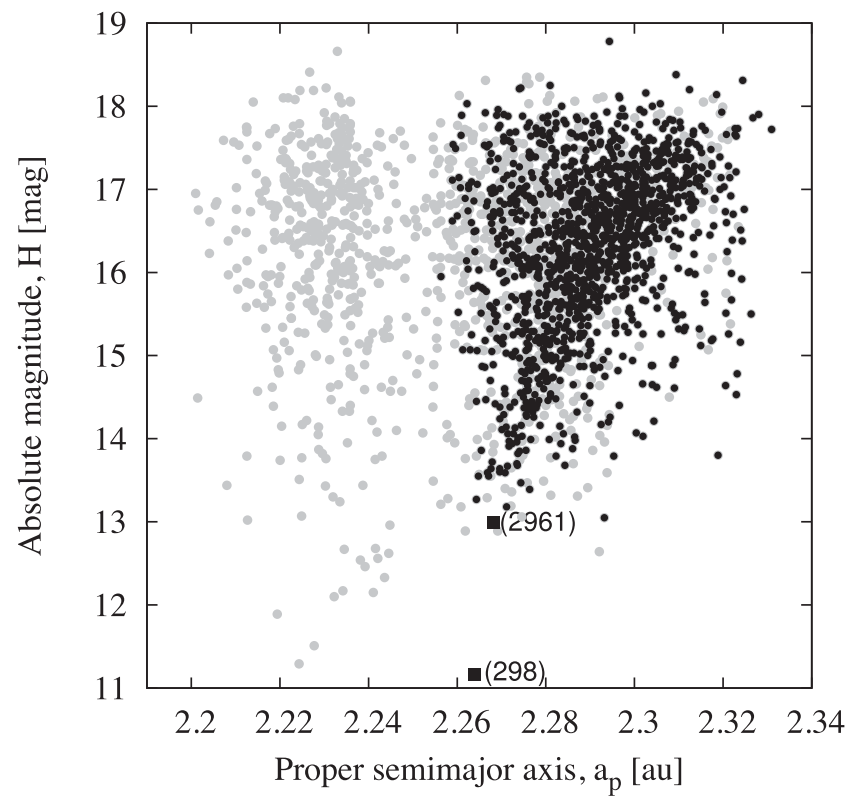

Figure 12. The distribution of the Baptistina (grey dots) and Levin (black dots) family members in the $a-H$ plane. A one-sided $\mathrm{V}$ shape of the Levin group seems to be the most dense region of the outer part of the Baptistina family V shape. This may suggest a common origin of the two groups. Note that asteroid (2961) Katsurahama is the largest member of the Levin dynamical group.

It is interesting to note that a part of the $\mathrm{V}$ shape is also visible on the left side, for absolute magnitudes above about 15.5, in agreement with results by Milić Žitnik \& Novaković (2015), who found that most of the objects in this magnitude range should be able to cross the $7 / 2$ resonance with Jupiter.

In summary, the Levin and Baptistina may belong to the same collisional family. Still, as described by Milani et al. (2014), it might be challenging to explain the current shape of this group in the proper elements space starting from fragments produced in a single collisional event. It should be noted, however, that this complexity depends on the cut-off distance used to identify the family, and could be avoided by adopting different, typically slightly larger, cut-off distances. Therefore, although there are some reasons to believe that Levin and Baptistina belong to the same collisional family, this question remains open. In this respect, our approach of interloper removal does not help much.

\subsection{Other families}

In this section we briefly discussed some of the results obtained for other families, focusing on the definition of the most appropriate cut-off value, i.e. the so-called nominal cut-off (see Table 3 ).

In most cases the nominal cut-off value is determined using the standard approach, i.e. the centre of a plateau (see Fig. 13). For some families the methodology was somewhat modified, as discussed below.

(20) Massalia: the number of asteroids associated with this family is steadily increasing with cut-off distance, until $40 \mathrm{~m} \mathrm{~s}^{-1}$ when the family merges with a local background population, and the number of associated objects jumps dramatically (see Fig. 13). Therefore, the nominal cut-off should be between 20 and $35 \mathrm{~m} \mathrm{~s}^{-1}$. We adopt a cut-off of $30 \mathrm{~m} \mathrm{~s}^{-1}$ to define the Massalia family, mainly because the $\mathrm{V}$ shape is well defined in this case. The number of identified 
Table 3. Nominal results for selected families. The columns are (1) family name (Family), (2) nominal cut-off value $\left(d_{\text {nom }}\right),(3)$ number of asteroids initially associated with a family at nominal cut-off (\# initial), (4) final number of asteroids associated with a family at nominal cut-off (\# final) and (5) percentage of removed asteroids (per cent).

\begin{tabular}{lcccr}
\hline Family & $d_{\text {nom }}$ & \#initial & \#final & $\%$ \\
\hline (5) Astraea & 45 & 4390 & 3915 & 10.8 \\
(10) Hygiea & 55 & 4272 & 4234 & 0.8 \\
(15) Eunomia & 55 & 10042 & 8128 & 19.1 \\
(20) Massalia & 30 & 4663 & 4648 & 0.3 \\
(24) Themis & 55 & 4076 & 3996 & 1.9 \\
(135) Hertha & 35 & 10901 & 9813 & 10.0 \\
(145) Adeona & 45 & 1994 & 1854 & 7.0 \\
(158) Koronis & 40 & 5644 & 5613 & 0.5 \\
(170) Maria & 55 & 2229 & 2146 & 3.7 \\
(221) Eos & 35 & 3352 & 3272 & 2.4 \\
(490) Veritas & 25 & 1168 & 1156 & 1.0 \\
(668) Dora & 40 & 1243 & 1234 & 0.7 \\
(847) Agnia & 30 & 2125 & 2110 & 0.7 \\
(1040) Klumpkea & 60 & 1739 & 1568 & 9.8 \\
(1272) Gefion & 50 & 2541 & 2306 & 9.2 \\
(1726) Hoffmeister & 35 & 1692 & 1686 & 0.4 \\
(2076) Levin & 45 & 2500 & 2346 & 6.2 \\
\hline
\end{tabular}

interlopers is consistent with a number of expected interlopers estimated by Migliorini et al. (1995).

(145) Adeona: similarly as in the case of the Massalia family, there is no clear plateau for the Adeona family in Fig. 13, although the number of associated asteroids grows slowly from 35 to $50 \mathrm{~m} \mathrm{~s}^{-1}$. The absence of a clear plateau for this family may be due to the close encounters with massive asteroids that significantly affected the dynamical evolution of the Adeona family (Carruba et al. 2003).

At $55 \mathrm{~m} \mathrm{~s}^{-1}$ the number of linked asteroids increases by factor of a few, and this is because the Adeona merges with the larger Eunomia family as discussed above. At this cut-off the family also extends on the outer side of the nearby $8 / 3$ resonance with Jupiter, located at $a=2.705 \mathrm{au}$. Examining the V shape of Adeona family, it seems that some family members should indeed be found on this side of the 8/3 resonance. However, due to proximity of the Eunomia family the nominal cut-off should be selected below $55 \mathrm{~m} \mathrm{~s}^{-1}$, and consequently possible members at $a>2.705$ au are lost. We adopt the membership found at $45 \mathrm{~m} \mathrm{~s}^{-1}$ as the nominal definition of the family.

(221) Eos: this family is unique from many aspects. The albedos of family members cover a range of values somewhere in between the typical values of $C$ and $S$ type, with an average albedo of $\overline{p_{v}}=$ $0.13 \pm 0.04$. The obtained $\overline{p_{v}}$ falls in the ambiguity interval (see Section 2.2.2). Hence, the albedo confidence interval for the Eos family needs to be set manually, and we adopt the $0.1-0.25$ range.

In the space of proper orbital elements the Eos family is located very close to the Veritas family ${ }^{14}$, and this complicates determination of its membership. These two families merge at cut-off of $40 \mathrm{~m} \mathrm{~s}^{-1}$, meaning that lower distance should be used to separate the two groups. However, at $35 \mathrm{~m} \mathrm{~s}^{-1}$ almost a half of the Eos family is missing. This is illustrated in Fig. 14. Therefore, a kind of artificial distinction between the Eos and Veritas family is necessary in order

\footnotetext{
${ }^{14}$ According to Michel et al. (2011), asteroid (490) Veritas might be an interloper in a family named after it. Therefore, the largest member of this family is (1086) Nata (see also Carruba, Vokrouhlický \& Nesvorný 2017).
}

to reasonably define the membership of both of the groups. Still, it should be noted that a smaller fraction of the Eos family, with $H>15$ mag and $a>3.156$ au, overlaps the Veritas family, suggesting that the latter may be contaminated by some members of the former group. Also, as noted by Tsirvoulis et al. (2017), members of the Eos family could be found at $a<2.95$ au, but a too high cut-off is needed to jump across the $7 / 3$ resonance with Jupiter that limited the family at the inner edge.

\section{ASTEROID FAMILIES PORTAL}

The large size and complexity of data acquired in the recent years create a demand for the new tools necessary to analyse this unprecedented flow of data. This is because it is often difficult to gather, manage and analyse all these data. One of the ways scientists are using to cope with these new challenges is the development of open access web portals. Such portals, typically devoted to specific purposes, allow scientists to easily review and analyse a large amount of available data.

In this work, we developed and launched this kind of a portal devoted to asteroid families. The portal is called Asteroid Families Portal (AFP) and is available at the following link: http://asteroids.matf.bg.ac.rs/fam/. The screenshot of the AFP home page is shown in Fig. 15.

The aim of the AFP is to collect different data about asteroid families and make these freely available to all interested researchers around the world. It can be used to quickly assess and visualized data. Still, this should not be the only purpose of this portal, but it should also allow us to apply different tools and methods to the provided data. For instance, the well-known HCM could be employed online to obtain the most recent list of the family members. Similarly, the method presented in this work could be used to produce a list of potential interlopers among family members.

A general idea of the AFP is to have two levels of the functionality for all the available tools, the automatic mode and the advance mode. The automatic mode is developed to produce the results using the most widely adopted settings and standards, and is mainly devoted to scientists who are not specialists in families. The advanced mode, on the other hand, offers to adjust many parameters used in the computations, and is primarily devoted to asteroid families specialist.

The AFP is foreseen to be continuously updated and upgraded. The current data sets will be updated frequently by adding newly available data. Among the next tools, we foresee for instance to offer a fully automatic estimation of family age, based on the so-called V-shaped method.

We also aim to develop an algorithm that will provide different basic information about an asteroid family, such as the size of the parent body and its corresponding escape velocity, the slope of the magnitude-frequency distribution, etc.

\section{CONCLUSIONS}

In this work, we present an automatic approach to exclude interlopers from the asteroid families. This approach combines the available data about spectral reflectance characteristics of asteroids and the iterative application of the HCM. This algorithm shows very promising characteristics and some advantages with respect to previously used techniques. The two most important improvements with respect to previous methods are the reduction of the chaining effect, the well-known drawback of the HCM and the fully automatic application of our method, freely available online at the AFP. 

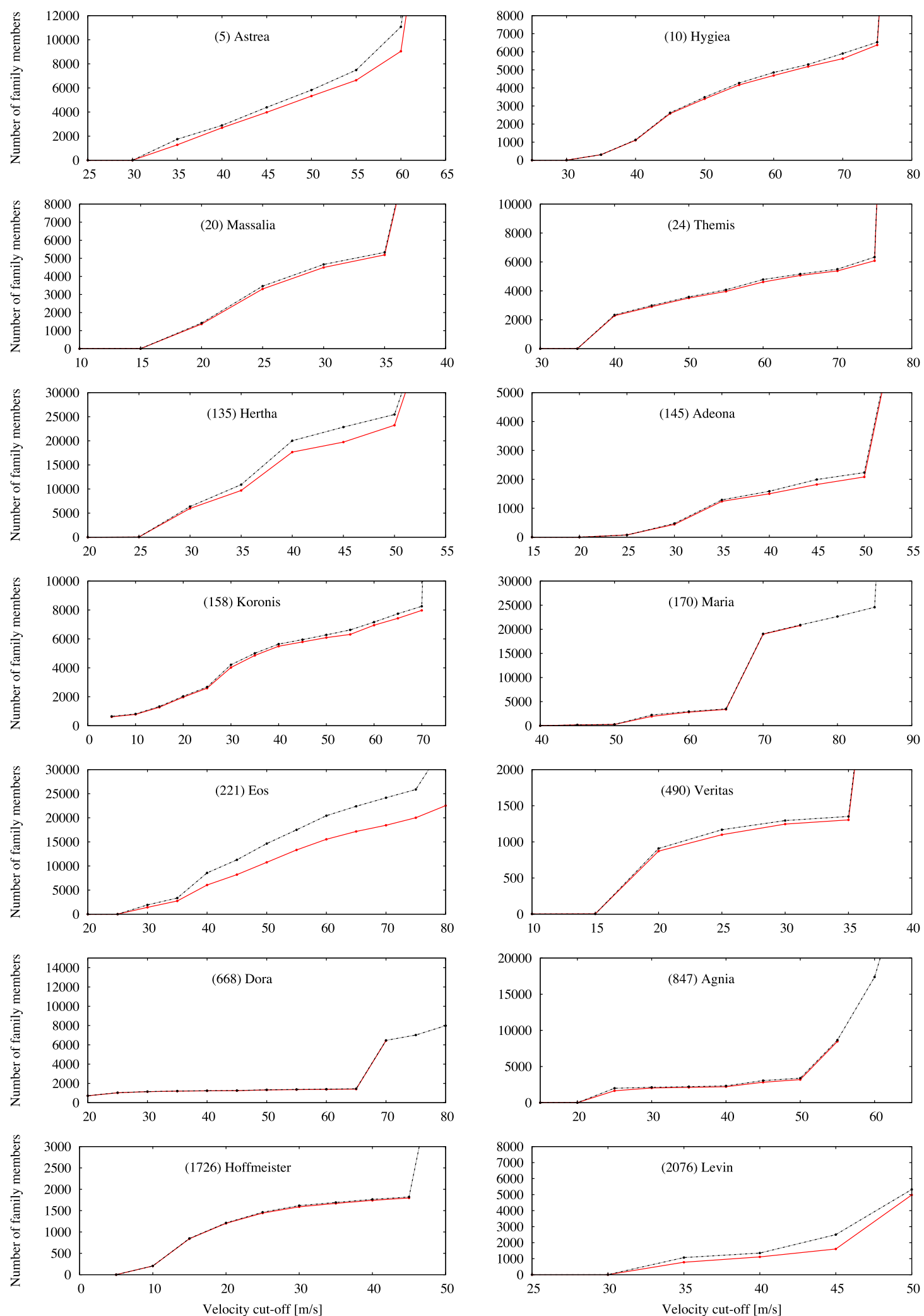

Figure 13. Number of asteroids associated with selected families using initial (dashed line) and modified (final) catalogues (solid line).

There are different possibilities to improve the presented method. Basically two directions could be followed: (i) to refine the way that we are using the existing data or (ii) to include new data. Regarding the first direction, an option definitely would be to try discriminate between different spectral types, not only between the $C$ and $S$ complexes. It is known that SDSS data, as used here, do not allow us to distinguish between the $C$ and $X$ type, while with the albedo data we cannot separate the $S$ from the $X$ type. However, taken 


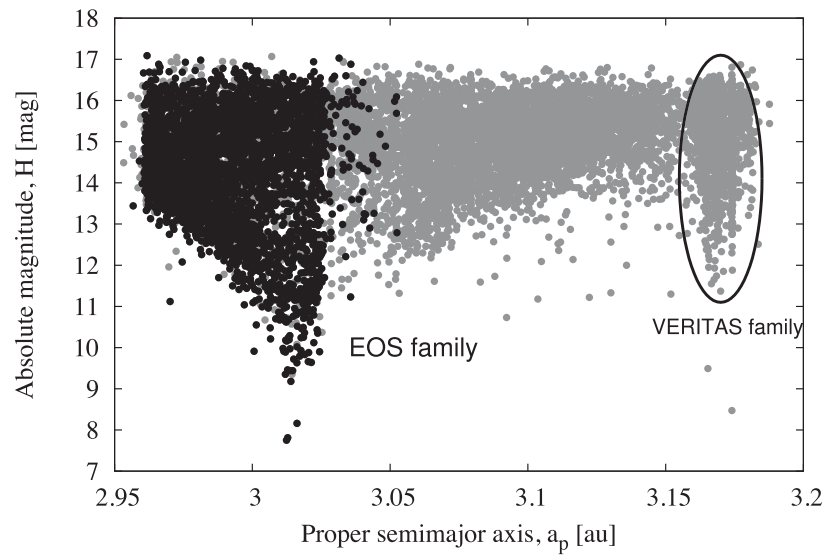

Figure 14. The distribution of the Eos family members in the $a-H$ plane. Black points represent family as identified at $35 \mathrm{~m} \mathrm{~s}^{-1}$, while grey points show members added at $40 \mathrm{~m} \mathrm{~s}^{-1}$.

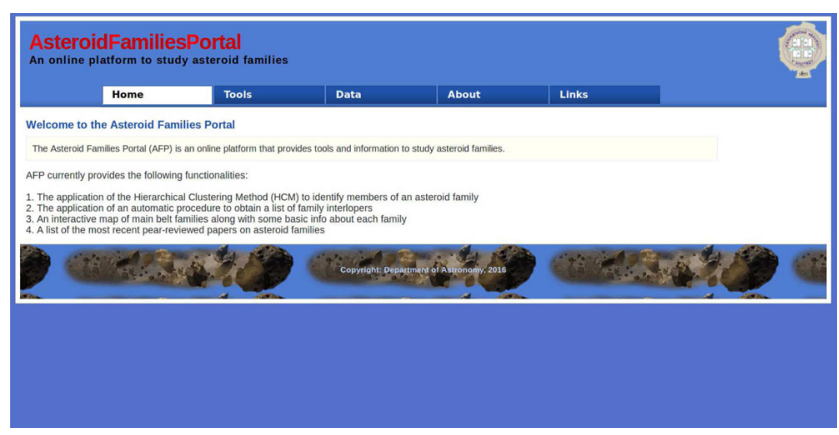

Figure 15. The home page of the AFP.

together this information would allow us to distinguish between the $C, S$ and $X$ type. Another option would be to use SDSS-based classification proposed by DeMeo \& Carry (2013), which makes possible even finer distinction between the asteroid spectral types.

There are also other data that may be useful to identify interlopers among the asteroid families. For instance, these include the taxonomy based on the new magnitude system as proposed by Oszkiewicz et al. (2012), or polarimetric data that were recently shown to be very useful to improve taxonomic classification (Belskaya et al. 2017).

Finally, our method should benefit a lot from the forthcoming GAIA mission data (Mignard et al. 2007; Warell \& Lagerkvist 2007). According to Delbo' et al. (2012), for all asteroids observed during the GAIA mission, it should be possible to perform multicolour photometry of quality good enough for a robust spectral classification.

\section{ACKNOWLEDGEMENTS}

The authors would like to thank Alberto Celino, the referee, for his valuable comments that helped to improve the manuscript. This work has been supported by the Ministry of Education, Science and Technological Development of the Republic of Serbia, under the Project 176011. VC acknowledges the support of the São Paulo State Science Foundation (FAPESP, grant 16/04476-8) and of the Brazilian National Research Council (CNPq, grant 305453/2011-4).

\section{REFERENCES}

Belskaya I. N. et al., 2017, Icarus, 284, 30

Bendjoya P., Slezak E., Froeschle C., 1991, A\&A, 251, 312

Bolin B. T., Delbo M., Morbidelli A., Walsh K. J., 2017, Icarus, 282, 290

Bottke W. F. et al., 2015, Icarus, 247, 191

Brouwer D., 1951, AJ, 56, 9

Brož M., Morbidelli A., Bottke W. F., Rozehnal J., Vokrouhlický D., Nesvorný D., 2013, A\&A, 551, A117

Bus S. J., 1999, PhD thesis, Massachusetts Institute of Technology

Bus S. J., Binzel R. P., 2002, Icarus, 158, 146

Carruba V., Burns J. A., Bottke W., Nesvorný D., 2003, Icarus, 162, 308

Carruba V., Domingos R. C., Nesvorný D., Roig F., Huaman M. E., Souami D., 2013, MNRAS, 433, 2075

Carruba V., Nesvorný D., Marchi S., Aljbaae S., 2016, MNRAS, 458, 1117

Carruba V., Vokrouhlický D., Nesvorný D., 2017, MNRAS, 469, 4400

Carvano J. M., Hasselmann P. H., Lazzaro D., Mothé-Diniz T., 2010, A\&A, 510, A43

Cellino A., Zappala V., 1993, Celest. Mech. Dyn. Astron., 57, 37

Cellino A., Zappalà V., Doressoundiram A., Di Martino M., Bendjoya P., Dotto E., Migliorini F., 2001, Icarus, 152, 225

Cellino A., Bus S. J., Doressoundiram A., Lazzaro D., 2002, in Bottke W. F., Cellino A., Paolicchi P., Binzel R. P., eds, Asteroids III. Univ. Arizona Press, Tucson, AZ

Cibulková H., Brož M., Benavidez P. G., 2014, Icarus, 241, 358

Delbo' M., Gayon-Markt J., Busso G., Brown A., Galluccio L., Ordenovic C., Bendjoya P., Tanga P., 2012, Planet. Space Sci., 73, 86

DeMeo F. E., Carry B., 2013, Icarus, 226, 723

DeMeo F. E., Binzel R. P., Slivan S. M., Bus S. J., 2009, Icarus, 202, 160

Dykhuis M. J., Greenberg R., 2015, Icarus, 252, 199

Hasegawa S., Müller T. G., Kuroda D., Takita S., Usui F., 2013, PASJ, 65 , 34

Hsieh H. H. et al., 2015, ApJ, 800, L16

Ivezić Ž. et al., 2001, AJ, 122, 2749

Ivezić Ž. et al., 2002, AJ, 124, 2943

Knežević Z., Milani A., 2000, Celest. Mech. Dyn. Astron., 78, 17

Lazzaro D. et al., 1999, Icarus, 142, 445

Mainzer A. et al., 2011a, ApJ, 731, 53

Mainzer A. et al., 2011b, ApJ, 741, 90

Masiero J. R. et al., 2011, ApJ, 741, 68

Masiero J. R., Mainzer A. K., Grav T., Bauer J. M., Jedicke R., 2012, ApJ, 759,14

Masiero J. R., Mainzer A. K., Bauer J. M., Grav T., Nugent C. R., Stevenson R., 2013, ApJ, 770, 7

Michel P., Jutzi M., Richardson D. C., Benz W., 2011, Icarus, 211, 535

Migliorini F., Zappalà V., Vio R., Cellino A., 1995, Icarus, 118, 271

Mignard F. et al., 2007, Earth Moon Planets, 101, 97

Milani A., Cellino A., Knežević Z., Novaković B., Spoto F., Paolicchi P., 2014, Icarus, 239, 46

Milani A., Spoto F., Knežević Z., Novaković B., Tsirvoulis G., 2016, in Chesley S. R., Morbidelli A., Jedicke R., Farnocchia D., eds, Proc. IAU Symp. 318, Asteroids: New Observations, New Models. Cambridge Univ. Press, Cambridge, p. 28

Milani A., Knežević Z., Spoto F., Cellino A., Novaković B., Tsirvoulis G., 2017, Icarus, 288, 240

Milić Žitnik I., Novaković B., 2015, MNRAS, 451, 2109

Nesvorný D., Vokrouhlický D., 2006, AJ, 132, 1950

Nesvorný D., Jedicke R., Whiteley R. J., Ivezić Ž., 2005, Icarus, 173, 132

Nesvorný D., Brož M., Carruba V., 2015, in Michel P., DeMeo F. E., Bottke W. F., eds, Asteroids IV. Univ. Arizona Press, Tucson, AZ

Novaković B., Cellino A., Knežević Z., 2011, Icarus, 216, 69

Novaković B., Hsieh H. H., Cellino A., Micheli M., Pedani M., 2014, Icarus, 231,300

Novaković B., Maurel C., Tsirvoulis G., Knežević Z., 2015, ApJ, 807, L5

Novaković B., Tsirvoulis G., Granvik M., Todović A., 2017, AJ, 153, 266

Oszkiewicz D. A., Bowell E., Wasserman L. H., Muinonen K., Penttilä A., Pieniluoma T., Trilling D. E., Thomas C. A., 2012, Icarus, 219, 283 
Parker A., Ivezić Ž., Jurić M., Lupton R., Sekora M. D., Kowalski A., 2008, Icarus, 198, 138

Rivkin A. S., Asphaug E., Bottke W. F., 2014, Icarus, 243, 429

Spoto F., Milani A., Knežević Z., 2015, Icarus, 257, 275

Tedesco E. F., Noah P. V., Noah M., Price S. D., 2002, AJ, 123, 1056

Tholen D. J., 1984, PhD thesis, Univ. Edinburgh

Tsirvoulis G., Novaković B., 2016, Icarus, 280, 300

Tsirvoulis G., Morbidelli A., Delbo M., Tsiganis K., 2017, Icarus, in press

Usui F. et al., 2011, PASJ, 63, 1117

Vokrouhlický D., Brož M., Bottke W. F., Nesvorný D., Morbidelli A., 2006, Icarus, 182,118

Vokrouhlický D., Bottke W. F., Chesley S. R., Scheeres D. J., Statler T. S., 2015, in Michel P., DeMeo F.E., Bottke W.F., eds, Asteroids IV. Univ. Arizona Press, Tucson, AZ
Walsh K. J., Delbó M., Bottke W. F., Vokrouhlický D., Lauretta D. S., 2013, Icarus, 225, 283

Warell J., Lagerkvist C.-I., 2007, A\&A, 467, 749

York D. G. et al., 2000, AJ, 120, 1579

Zappala V., Farinella P., Knežević Z., Paolicchi P., 1984, Icarus, 59, 261

Zappala V., Cellino A., Farinella P., Knežević Z., 1990, AJ, 100, 2030

Zappala V., Cellino A., Farinella P., Milani A., 1994, AJ, 107, 772

Zappalà V., Bendjoya P., Cellino A., Farinella P., Froeschlé C., 1995, Icarus, 116, 291

This paper has been typeset from a $\mathrm{T}_{\mathrm{E}} \mathrm{X} / \mathrm{LT} \mathrm{E} \mathrm{X}$ file prepared by the author. 